\title{
Advancing the global public health agenda for NAFLD: a consensus statement
}

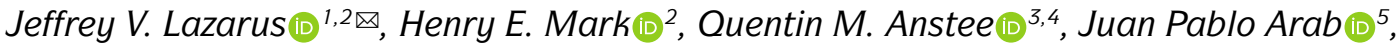
Rachel L. Batterham 6 , Laurent Castera $\mathbb{1}^{7}$, Helena Cortez-Pinto $\mathbb{1}^{8}$, Javier Crespo ${ }^{9}{ }^{9}$, Kenneth Cusi ${ }^{10}$, M. Ashworth Dirac ${ }^{11}$, Sven Francque ${ }^{12,13}$, Jacob George ${ }^{14}{ }^{14}$, Hannes Hagström ${ }^{15}$, Terry T.-K. Huang ${ }^{16}{ }^{16}$, Mona H. Ismail(1) ${ }^{17}$, Achim Kautz ${ }^{18}$, Shiv Kumar Sarin (10 ${ }^{19}$, Rohit Loomba (i) ${ }^{20,21}$, Veronica Miller ${ }^{22}$, Philip N. Newsome ${ }^{23}$, Michael Ninburg ${ }^{24}$, Ponsiano Ocama ${ }^{25}$, Vlad Ratziu ${ }^{26}$, Mary Rinella ${ }^{27}$, Diana Romero ${ }^{28}$, Manuel Romero-Gómez $\mathbb{1}^{29}$, Jörn M. Schattenberg $\mathbb{1}^{30}$, Emmanuel A. Tsochatzis ${ }^{31,32}$, Luca Valenti33,34, Vincent Wai-Sun Wong ${ }^{30}{ }^{35}$, Yusuf Yilmaz $\mathbb{1}^{36,37}$, Zobair M. Younossi ${ }^{38}$, Shira Zelber-Sagi ${ }^{39,40}$ and the NAFLD Consensus Consortium*

Abstract | Non-alcoholic fatty liver disease (NAFLD) is a potentially serious liver disease that affects approximately one-quarter of the global adult population, causing a substantial burden of ill health with wide-ranging social and economic implications. It is a multisystem disease and is considered the hepatic component of metabolic syndrome. Unlike other highly prevalent conditions, NAFLD has received little attention from the global public health community. Health system and public health responses to NAFLD have been weak and fragmented, and, despite its pervasiveness, NAFLD is largely unknown outside hepatology and gastroenterology. There is only a nascent global public health movement addressing NAFLD, and the disease is absent from nearly all national and international strategies and policies for non-communicable diseases, including obesity. In this global Delphi study, a multidisciplinary group of experts developed consensus statements and recommendations, which a larger group of collaborators reviewed over three rounds until consensus was achieved. The resulting consensus statements and recommendations address a broad range of topics - from epidemiology, awareness, care and treatment to public health policies and leadership — that have general relevance for policy-makers, health-care practitioners, civil society groups, research institutions and affected populations. These recommendations should provide a strong foundation for a comprehensive public health response to NAFLD.

Non-alcoholic fatty liver disease (NAFLD) is a potentially serious liver condition that, on a societal level, results in substantial health-care costs, economic losses and reduced health-related quality of life (HRQoL) $)^{1-8}$. A biologically and clinically heterogeneous disease, NAFLD covers a broad spectrum of histological conditions that increase both hepatic and non-hepatic morbidity and mortality. The majority of people living with NAFLD have isolated steatosis (non-alcoholic fatty liver, NAFL) and a smaller proportion develop non-alcoholic steatohepatitis (NASH), with increasing hepatic fibrosis leading eventually to cirrhosis, liver cancer, end-stage liver disease and death ${ }^{9,10}$. NASH is a leading cause of progression to cirrhosis and hepatocellular carcinoma ${ }^{11,12}$, and an increasingly common indication for liver transplantation ${ }^{13,14}$. Liver cancer is now the second leading cause of years of life lost among all cancers globally ${ }^{15}$.

NAFLD is part of a multisystem disease and is considered the hepatic manifestation of metabolic syndrome ${ }^{16-18}$. Although strongly associated with obesity, NAFLD also occurs in individuals with normal weight, especially in Asian populations ${ }^{19,20}$. The causes of death in people living with NAFLD vary depending on disease state. Patients with cirrhosis predominantly have liver-related events, whereas those without cirrhosis have vascular events and non-hepatic cancer ${ }^{21}$. Overall, cardiovascular disease (CVD) is the leading cause of death in patients with NAFLD; other common causes include extrahepatic 
malignancies, liver-related complications, chronic kidney disease and type 2 diabetes mellitus (T2DM) ${ }^{16,17,22-24}$.

NAFLD is closely related to other highly prevalent non-communicable diseases (NCDs) with substantial overlap in the public health and health system approaches needed to prevent and manage these conditions. However, NAFLD is currently absent from major global and national NCD strategies and action plans ${ }^{25,26}$, and efforts to integrate NAFLD into the NCD agenda have been minimal. Despite the scale of the challenge and the human, social and economic implications of the disease, few people outside the fields of hepatology and gastroenterology are familiar with NAFLD, and there is no global public health movement to address the disease.
In this Consensus Statement, a global multidisciplinary group of experts developed consensus statements and recommendations for tackling the burden of NAFLD. The overarching goal was to develop a foundation for comprehensive public health responses to NAFLD and to outline catalytic actions that will move this agenda forwards in the coming years. Using a Delphi-based approach, the Consensus Statement sets out current thinking on NAFLD in areas ranging from epidemiology, awareness, care and treatment to public health policies and leadership (FIG. 1). The consensus statements and recommendations should have broad relevance for policy-makers, health-care practitioners, civil society groups, research institutions and affected populations.

\footnotetext{
Author addresses

${ }^{1}$ Barcelona Institute for Global Health (ISGlobal),

Hospital Clínic, University of Barcelona, Barcelona, Spain.

${ }^{2}$ EASL International Liver Foundation, Geneva, Switzerland.

${ }^{3}$ Translational \& Clinical Research Institute, Faculty of

Medical Sciences, Newcastle University, Newcastle upon

Tyne, UK.

${ }^{4}$ Newcastle NIHR Biomedical Research Centre, Newcastle upon Tyne Hospitals NHS Trust, Newcastle upon Tyne, UK.

${ }^{5}$ Departamento de Gastroenterologia, Escuela de

Medicina, Pontificia Universidad Catolica de Chile,

Santiago, Chile.

${ }^{6}$ Centre for Obesity Research, University College

London and National Institute of Health Research,

UCLH Biomedical Research Centre, London, UK.

${ }^{7}$ Department of Hepatology, Hôpital Beaujon, Université de

Paris, Paris, France.

${ }^{8}$ Clínica Universitária de Gastrenterologia, Laboratório de

Nutrição, Faculdade de Medicina, Universidade de Lisboa,

Lisbon, Portugal.

${ }^{9}$ Gastroenterology and Heptology Unit, Hospital

Universitario Marqués de Valdecilla, Santander, IDIVAL,

Santander, Spain.

${ }^{10}$ Division of Endocrinology, Diabetes \& Metabolism,

Department of Medicine, Veterans Health Administration

and University of Florida, Gainesville, FL, USA.

${ }^{11}$ Department of Health Metrics Sciences, Department

of Family Medicine, University of Washington, Seattle,

WA, USA.

${ }^{12}$ Department of Gastroenterology Hepatology,

University Hospital Antwerp, Antwerp, Belgium.

${ }^{13}$ Translational Sciences in Inflammation and Immunology

TWI2N, Faculty of Medicine and Health Sciences,

University of Antwerp, Antwerp, Belgium.

${ }^{14}$ Storr Liver Centre, Westmead Institute for Medical

Research, Westmead Hospital and University of Sydney,

Sydney, New South Wales, Australia.

${ }^{15}$ Department of Medicine, Karolinska Institutet,

Stockholm, Sweden.

${ }^{16}$ Center for Systems and Community Design and

NYU-CUNY Prevention Research Center, Graduate

School of Public Health and Health Policy, City University

of New York, New York, NY, USA.

${ }^{17}$ Division of Gastroenterology, Department of Internal

Medicine, King Fahd Hospital of the University, College

of Medicine, Imam Abdulrahman Bin Faisal University,

Dammam, Saudi Arabia.

${ }^{18}$ Kautz5, Köln, Germany.

${ }^{19}$ Department of Hepatology, Institute of Liver and

Biliary Sciences, New Delhi, India.

${ }^{20}$ Department of Medicine, NAFLD Research Center,

La Jolla, CA, USA.

${ }^{21}$ Department of Medicine, University of California San Diego, La Jolla, CA, USA.

${ }^{22}$ University of California Berkeley, School of Public Health, Forum for Collaborative Research, Washington, DC, USA.

${ }^{23}$ National Institute for Health Research Birmingham

Biomedical Research Centre at University Hospitals

Birmingham NHS Foundation Trust and the University

of Birmingham, Birmingham, UK.

${ }^{24}$ Hepatitis Education Project, Seattle, WA, USA.

${ }^{25}$ Department of Medicine, College of Health Sciences,

Makerere University, Kampala, Uganda.

${ }^{26}$ Assistance Publique-Hôpitaux de Paris, Hôpital

Pitie-Salpetriere, University of Paris, Paris, France.

${ }^{27}$ Department of Medicine, Feinberg School of Medicine,

Northwestern University, Chicago, IL, USA.

${ }^{28}$ Department of Community Health and Social Sciences,

CUNY Graduate School of Public Health and Health Policy,

New York, NY, USA.

${ }^{29}$ UCM Digestive Diseases, CIBEREHD and IBIS, Virgen

del Rocío University Hospital, University of Seville,

Seville, Spain.

${ }^{30}$ Metabolic Liver Research Program, I. Department of Medicine, University Medical Centre Mainz, Mainz, Germany.

${ }^{31}$ University College London Institute for Liver and

Digestive Health, Royal Free Hospital, London, UK.

${ }^{32}$ Sheila Sherlock Liver Centre, Royal Free Hospital,

London, UK.

${ }^{33}$ Department of Pathophysiology and Transplantation,

Università degli Studi di Milano, Milan, Italy.

${ }^{34}$ Precision Medicine, Department of Transfusion Medicine and Hematology, Fondazione IRCCS Ca' Granda Ospedale Maggiore Policlinico, Milan, Italy.

${ }^{35}$ Department of Medicine and Therapeutics, The Chinese University of Hong Kong, Hong Kong, China.

${ }^{36}$ Department of Gastroenterology, Marmara University

School of Medicine, Istanbul, Turkey.

${ }^{37}$ Liver Research Unit, Institute of Gastroenterology,

Marmara University, Istanbul, Turkey.

${ }^{38}$ Center for Liver Diseases, Inova Medicine, Falls Church,

VA, USA.

${ }^{39}$ University of Haifa, Faculty of Social Welfare and

Health Sciences, School of Public Health, Mount Carmel,

Haifa, Israel.

${ }^{40}$ Department of Gastroenterology, Tel-Aviv Medical

Centre, Tel-Aviv, Israel.

*A list of authors and their affiliations appears at the end of the paper.
} 


\section{CONSENSUS STATEMENT}

a

December 2020

\begin{tabular}{l|l}
$\begin{array}{l}\text { Core group } \\
\text { - Core group } \\
\text { of } 33 \text { draft } \\
\text { consensus } \\
\text { statements }\end{array}$ & $\begin{array}{l}\text { Consortium } \\
-218 \text { collaborators }\end{array}$ \\
\hline & \\
\end{tabular}

b

Leadership for the NAFLD public health agenda

- Form a global coalition

to develop a roadmap

- Collaborate across

disciplines

- Develop guidelines, policy

briefs and action plans
January-February 2021

Delphi Round 1 and 2

R1

- 38 statements; mean agreement $=80 \%$

R2

- 37 statements; mean

agreement $=91 \%$
16 February 2021

Online convening

of core group

- Review responses

to R1 and R2

and draft

recommendations
February 2021

Web review

of draft

recommendations

- Consortium

members

feedback on draft

recommendations
March 2021

Delphi round 3

- 37 statements;

mean agreement $=99 \%$

- 26 recommendations;

mean agreement $=98 \%$

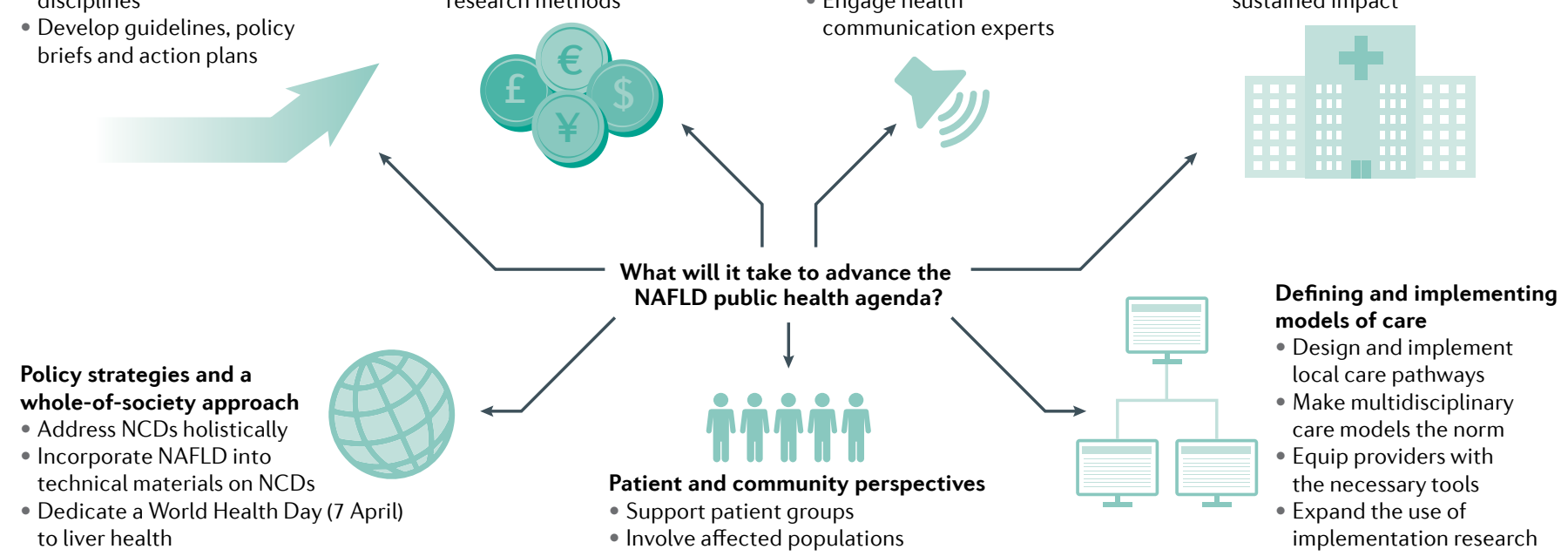

Human and economic burden

- Invest in research

- Develop global, regional and

local investment cases

- Consider alternate

research methods

\section{Awareness}

- Reconsider the terminology of fatty liver diseases

- Develop simple knowledge products and educational courses

- Engage health

communication experts
- Involve affected populations
Treatment and care

- Improve access to effective treatments

- Standardize trial end points

- Identify interventions with sustained impact

Fig. 1 | Development of the NAFLD consensus statements and recommendations. a | The development process for the development of the non-alcoholic fatty liver disease (NAFLD) consensus statement and recommendations following a Delphi methodology. $\mathbf{b}$ | Summary of the 26 recommendations stemming from this process. NCDs, non-communicable diseases.

\section{Methods}

\section{Expert panel members and topics}

A core group of 33 experts (TABLE 1) were identified by the European Association for the Study of the Liver (EASL) International Liver Foundation (EILF) to lead a Delphi study to develop consensus statements and recommendations to advance the NAFLD public health agenda. The chair (J.V.L.) and project coordinator (H.E.M.) led this group of clinicians, researchers, advocates, academics and civil society experts from 16 countries through the development and implementation of the Delphi process. Core group members identified additional experts to be invited to participate in the Delphi consensus-building process. The final panel comprised 218 individuals, including the core group members. The demographic description of the expert panel is summarized in TABLE 2 and its geographical diversity in Supplementary Table 1.

In December 2020, the core group drafted the statements to be used for the initial Delphi survey round and the statements were grouped into the following categories: (1) human and economic burden; (2) awareness; (3) defining and implementing models of care, including (3a) general considerations, (3b) considerations for children and adolescents, and (3c) considerations for low-resource settings; (4) treatment and care; (5) patient and community perspectives; (6) policy strategies and a whole-of-society approach; and (7) leadership for the NAFLD public health agenda.

\section{Delphi method data collection}

The Delphi method design ${ }^{27}$ consisted of five components of data collection, including a first and second survey round ( $R 1$ in January 2021, R2 in February 2021), an online convening of the core group (16 February 2021), a web-based review of draft recommendations (February 2021), and a final survey round (R3 in March 2021). We used the Qualtrics XM platform to develop and distribute the surveys. The data collection periods for each survey round ranged between 1.5 and 3 weeks, allowing for holiday periods. The R1 survey contained $38 \mathrm{draft}$ statements with four-point Likert-type categories for respondents to indicate their level of agreement with the statements (that is, 'Agree'/'Somewhat agree'/'Somewhat disagree'/'Disagree'). In this round, respondents who agreed or somewhat agreed with a statement could provide comments and suggest edits, while those who disagreed or somewhat disagreed could explain why. The R2 survey contained 37 statements and reflected suggestions from R1, including new, revised and merged statements. In the R2 survey, we included text-box summaries of the edits made to each of the statements for respondents to consider as they indicated their level of agreement or 


\section{CONSENSUS STATEMENT}

Table 1 | Core group members $(\boldsymbol{n}=\mathbf{3 3})$

\begin{tabular}{|c|c|c|}
\hline Name & Affiliation & $\begin{array}{l}\text { Country/territory } \\
\text { where currently based }\end{array}$ \\
\hline Quentin M. Anstee & Newcastle University & UK \\
\hline Juan Pablo Arab & Pontifical Catholic University of Chile & Chile \\
\hline Rachel L. Batterham & University College London & UK \\
\hline Laurent Castera & University of Paris & France \\
\hline Helena Cortez Pinto & University of Lisbon & Portugal \\
\hline Javier Crespo & Universidad de Cantabria & Spain \\
\hline Kenneth Cusi & $\begin{array}{l}\text { Veterans Health Administration and University } \\
\text { of Florida }\end{array}$ & USA \\
\hline M. Ashworth Dirac & University of Washington & USA \\
\hline Sven Francque & Antwerp University Hospital & Belgium \\
\hline Jacob George & University of Sydney & Australia \\
\hline Hannes Hagström & Karolinska Institutet & Sweden \\
\hline Terry T.-K. Huang & $\begin{array}{l}\text { City University of New York Graduate School of Public } \\
\text { Health and Health Policy }\end{array}$ & USA \\
\hline \multirow[t]{2}{*}{ Mona H. Ismail } & $\begin{array}{l}\text { College of Medicine, Imam Abdulrahman Bin Faisal } \\
\text { University }\end{array}$ & Saudi Arabia \\
\hline & King Fahad Hospital of the University, Al-Khobar & \\
\hline Achim Kautz & Kautz $\mathrm{gUG}^{5}$ & Germany \\
\hline Shiv Kumar Sarin & Institute of Liver and Biliary Science & India \\
\hline Jeffrey V. Lazarus (Chair) & Barcelona Institute for Global Health (ISGlobal) & Spain \\
\hline Rohit Loomba & University of California, San Diego & USA \\
\hline Henry E. Mark (Project coordinator) & EASL International Liver Foundation & Switzerland \\
\hline Veronica Miller & University of California, Berkeley & USA \\
\hline Phil N. Newsome & University of Birmingham & UK \\
\hline Michael Ninburg & Hepatitis Education Project & USA \\
\hline Ponsiano Ocama & Makerere University College of Health Sciences & Uganda \\
\hline Vlad Ratziu & Sorbonne University & France \\
\hline Mary Rinella & Northwestern University Feinberg School of Medicine & USA \\
\hline Diana Romero (Non-voting) & $\begin{array}{l}\text { City University of New York Graduate School of Public } \\
\text { Health and Health Policy }\end{array}$ & USA \\
\hline Manuel Romero-Gómez & Virgen del Rocío University Hospital & Spain \\
\hline Jörn M. Schattenberg & University Medical Center Mainz & Germany \\
\hline Emmanuel Tsochatzis & UCL Institute for Liver and Digestive Health & UK \\
\hline Luca Valenti & University of Milan & Italy \\
\hline Vincent W. S. Wong & The Chinese University of Hong Kong & China \\
\hline Yusuf Yilmaz & Marmara University & Turkey \\
\hline Zobair M. Younossi & Inova Medicine, Inova Health System & USA \\
\hline Shira Zelber-Sagi & University of Haifa School of Public Health & Israel \\
\hline
\end{tabular}

disagreement with the statements. The open-ended comment options were again provided to all respondents except those who agreed with a given statement.

A majority of core group members (27 of 33) participated in the online convening following the R2 survey, which permitted in-depth breakout-group discussions on issues that arose in the first two rounds. This convening was hosted and facilitated by Wilton Park, a UK-based global forum for strategic dialogue. Concurrent with revising the statements for R3, the core group developed a draft set of recommendations to accompany the consensus statements. Preliminary feedback on these recommendations from the larger expert panel was sought over a 1-week period via a shared Google document. The resulting 26 recommendations were included with the final set of 37 statements in R3. Given fairly high levels of agreement in the previous survey rounds, the consensus statements and recommendations in R3 were presented with a binary ('Agree'/'Disagree') response option. A text box at the end of each of the survey domain sections provided respondents with the option to include final comments. 
Finally, we assigned each statement and recommendation a grade to indicate the level of agreement, utilizing a grading system recently used in other Delphi studies ${ }^{28,29}$ in which ' $U$ ' denotes unanimous (100\%) agreement, 'A' 90-99\% agreement, 'B' 78-89\% agreement, and 'C' $67-77 \%$ agreement.

\section{Findings}

Here, we report the final statements and recommendations along with a summary of the broader literature as it relates to them.

\section{Consensus statements and recommendations}

Across Delphi rounds there was a consistent increase in consensus for all statements. The mean percentage of 'Agree' responses rose from $80.3 \%$ in R1 to $90.9 \%$ in R2 and $98.5 \%$ in R3. The incorporation of substantive comments from respondents into the statements

\begin{tabular}{|c|c|}
\hline \multicolumn{2}{|c|}{$\begin{array}{l}\text { Table } 2 \text { I Expert panel demographic composition } \\
\text { and level of engagement }\end{array}$} \\
\hline Characteristic & Values \\
\hline Total & 218 \\
\hline \multicolumn{2}{|l|}{$G_{e n d e r}^{a}$} \\
\hline Man & $67.0 \%(146)$ \\
\hline Woman & $30.7 \%(67)$ \\
\hline Prefer not to say/no response & $2.3 \%(5)$ \\
\hline \multicolumn{2}{|l|}{ Primary sector of employment } \\
\hline Academic & $71.1 \%(155)$ \\
\hline Civil society & $4.1 \%(9)$ \\
\hline Public & $15.1 \%(33)$ \\
\hline Private & $4.6 \%(10)$ \\
\hline Other/no response & $5.0 \%(11)$ \\
\hline \multicolumn{2}{|l|}{ Primary field of employment } \\
\hline Health-care provider & $21.1 \%(46)$ \\
\hline Clinical research & $62.8 \%(137)$ \\
\hline Non-clinical research & $3.2 \%(7)$ \\
\hline Advocacy & $5.0 \%(11)$ \\
\hline Other/no response & $7.8 \%(17)$ \\
\hline \multicolumn{2}{|l|}{ Geographical representation } \\
\hline Countries/regions of origin ( $n$ ) & 89 \\
\hline Countries/regions currently based in ( $n$ ) & 91 \\
\hline \multicolumn{2}{|l|}{ Delphi process engagement ${ }^{b}$} \\
\hline Round 1 survey & $87.6 \%(191)$ \\
\hline Round 2 survey & $88.1 \%(192)$ \\
\hline World Café core group meeting & $81.8 \%(27)$ \\
\hline Round 3 survey & $84.9 \%(185)$ \\
\hline Participation in one or more components & 218 \\
\hline Mean no. of surveys engaged in & 2.05 \\
\hline
\end{tabular}

increased the level of support in subsequent survey rounds. In the end, there was unanimous agreement with 7 statements and $>90 \%$ agreement with another 30 statements (TABLE 3; translations for TABLE 3 are available in Arabic, Chinese (Mandarin), French, German, Italian and Spanish in Supplementary Tables 2-7). For the associated recommendations, the mean percentage of agreement for the 26 recommendations was $98 \%$. Three recommendations met with unanimous agreement, 22 others with $>90 \%$ agreement, and the final one with $>80 \%$ agreement (TABLE 4; translations for TABLE 4 are available in Arabic, Chinese (Mandarin), French, German, Italian and Spanish in Supplementary Tables 2-7).

\section{The human and economic burden \\ - Statements 1.1-1.6 \\ - Recommendations 1-3}

Epidemiology in adults. The global prevalence of NAFLD among adults is estimated to be $23-25 \%^{30,31}$. The burden varies between and within regions, with the highest prevalence in the Middle East (32\%) and South America (30\%) and the lowest in Africa (13\%) ${ }^{31}$. Up to $20 \%$ of people with NAFLD are affected by $\mathrm{NASH}^{31-33}$. However, there are few reliable epidemiological estimates disaggregated by fibrosis stage, age, gender and geographical location. The need for resource-intensive procedures to accurately assess and determine disease severity is a barrier to population-based surveillance for NAFLD, as is the variety of diagnostic methods and criteria. The availability of good quality data continues to hinder concerted national and global action on NAFLD

In most populations, the burden of NAFLD increases proportionally with increases in $\mathrm{BMI}^{34}$, although the condition is also common in individuals without overt metabolic risk factors ${ }^{19,35}$. In the vast majority of patients, NAFLD emerges in the context of metabolic syndrome, with insulin resistance an important pathophysiological mechanism ${ }^{17}$. NAFLD prevalence is higher among patients with T2DM than in the general population, whereas T2DM incidence is higher in patients with NAFLD $^{17,24,36,37}$. Driven by increasing prevalences of obesity and T2DM and by ageing populations, the global NAFLD burden is projected to grow in the coming decade $^{32,33}$.

Between 1990 and 2017, global deaths due to cirrhosis increased from 899,000 to 1.32 million while disability-adjusted life years increased from 30.5 million to 41.4 million. During this period, the number of prevalent cases of compensated cirrhosis due to NASH more than doubled, whereas for decompensated cirrhosis the figure more than tripled. With the expansion of prevention and treatment measures for hepatitis $B$ and $C$, NASH is expected to overtake them soon as the leading cause of cirrhosis ${ }^{38}$.

Epidemiology in children and adolescents. Epidemiological data on NAFLD in children and adolescents are scarce. There is marked heterogeneity in the findings of available studies, due in part to variations in 
Table 3 | Consensus statements for a NAFLD public health agenda

Number Statement

1. The human and economic burden

1.1 According to current estimates, 20-25\% of the global adult population is affected by NAFLD, and an estimated $20 \%$ of people with NAFLD will develop NASH. However, robust epidemiological estimates, disaggregated by fibrosis stage, age, gender, risk profile and geographical area, are limited. Incomplete data hinder concerted action at the national and global levels.

Data from central registries, electronic health-care records or official statistics are available for certain countries and can be useful sources of information. However, differences in reporting, including the use of different administrative codes (for example, the International Classification of Disease (ICD) codes), limit comparability.

1.3 Data on paediatric NAFLD are scarce. Prevalence estimates vary widely, whilst there is limited information on long-term health outcomes in children living with NAFLD. However, available data indicate that NAFLD is an increasing problem in paediatric populations and is especially prevalent in children with obesity.

1.4 A wide range of factors needs to be considered in developing prevention and treatment approaches for NAFLD. These factors extend from metabolic risks, including insulin resistance, to genetic, social and environmental influences that may play a part in the development and progression of the disease.

1.5 NAFLD shares a bidirectional relationship with other metabolic conditions. Addressing NAFLD will likely reduce the prevalence and severity of these conditions.

1.6 There are both economic and social arguments for taking action on NAFLD. Evidence shows that NAFLD progression is associated with substantial health-care costs, socioeconomic losses and reduced quality of life, most notably in patients with advanced fibrosis and cirrhosis. Early intervention could help reduce the burden of disease, associated health-care costs and economic losses.

\section{Awareness and education}

2.1 Communicating about NAFLD and its consequences has proved to be a major challenge for the liver health community.

2.2 Raising the profile of NAFLD as a public health issue will require clear messages about the condition, its consequences and what action is required. These messages should be tailored to specific audiences, including the liver and gastroenterology communities, primary care providers, specialists from other relevant disciplines, as well as stakeholders such as at-risk groups, the media and policy-makers.

2.3 Primary care providers and diabetes specialists can play a critical part in identifying and referring patients with advanced fibrosis to liver specialists. Raising the awareness of these medical providers would improve their ability to play this part.

\section{3a. Defining and implementing models of care: general considerations}

3.1 Given the broad disease spectrum of NAFLD and the different levels of care required by patients across this spectrum, having clearly defined, context-specific models of care will be important for addressing the disease burden.

3.2 The majority of people living with NAFLD can be managed in primary care; only patients with advanced disease need referral to a liver specialist. NAFLD care pathways can guide care decisions, including decisions on when to refer a patient to specialist care.

3.3 People living with NAFLD, especially those with advanced fibrosis, commonly require the management of multiple comorbid conditions, including diabetes, obesity and cardiovascular disease.

3.4 There is limited evidence on the impact of different NAFLD models of care on patient outcomes and cost-effectiveness. The lack of evidence and of investment in implementation research continues to impede the design and delivery of good care in different health-care settings and contexts.

3.5 Fibrosis stage is an important predictor of long-term liver-related outcomes and overall mortality in people living with NAFLD. Evidence of advanced fibrosis is an adequate indicator of a patient's need for referral to specialist liver care.

3.6 Non-invasive tests (NITs) can be effective at excluding advanced fibrosis and the need for further assessment or referral to specialist liver care, especially when combinations of NITs are used sequentially.

3.7 The availability and use of different NITs vary among health-care settings. Non-commercial blood-based scores could be feasibly implemented in most primary and secondary care settings, such as diabetes clinics, if they were more readily available and widely known.

3.8 People living with type 2 diabetes mellitus (T2DM) and/or obesity are recognized as being at high risk for NAFLD-related complications. Collaboration and coordination across the different components of the health-care system will be needed to care for these patients most effectively.

\section{3b. Defining models of care: considerations for children (younger than 18 years)}

3.9 The natural history of paediatric NAFLD is poorly understood, due to a lack of prospective studies and the complex nature of the disease, including pathologies that are unique to children living with NAFLD. Better data on the natural history, pathophysiology and risk factors for disease progression would improve the care of this population.

3.10 Models of care for children should address all care needs, including the provision of psychological support, and be designed to facilitate the smooth transfer of care from paediatric to adult services. 
Table 3 (cont.) | Consensus statements for a NAFLD public health agenda

\begin{tabular}{|c|c|c|}
\hline Number & Statement & Grade \\
\hline \multicolumn{3}{|c|}{ 3b. Defining models of care: considerations for children (younger than 18 years) (cont.) } \\
\hline 3.11 & The lack of validated NITs for use in children is a barrier to timely diagnosis and linkage to care. & A \\
\hline 3.12 & $\begin{array}{l}\text { Available data show that paediatric NAFLD is associated with both hepatic and non-hepatic } \\
\text { morbidity and mortality. Children living with NAFLD might benefit from multidisciplinary } \\
\text { management approaches tailored to their unique health-care needs. }\end{array}$ & A \\
\hline
\end{tabular}

3.13 In low-resource settings, the availability of diagnostic tools - including NITs - is likely to be A limited, especially the more expensive imaging-based tests. Diagnosis in these settings will often require practitioners to make pragmatic choices and resort to low-cost solutions.

3.14 NAFLD is not mentioned in the current guidelines from the WHO on the detection, diagnosis and treatment of major non-communicablediseases (NCDs) in primary care in low-resource settings. Inclusion of NAFLD in such guidance would help to improve care for affected populations in these settings.

\section{Treatment and care}

4.1 Interventions aimed at modifying lifestyle risk factors are the cornerstone of NAFLD treatment. There is some evidence that these interventions can prevent disease progression and can, in some cases, reverse fibrosis, yet more data will help to identity the most effective approaches and how to implement them in clinical practice.

4.2 As the number of effective pharmacological treatments for NAFLD increases, programmes aimed at modifying lifestyle risk factors will continue to be a core element of NAFLD disease management.

4.3 Access to treatment programmes for NAFLD requires that they be incorporated into relevant national health-care policies and guidelines and be adequately funded. Private and public payers and/or funders have a key part to play in ensuring financial support (for example, reimbursement) for these services.

4.4 The invasive nature of liver biopsy, the inherent variability of histological findings and the lack of an alternative validated surrogate for long-term clinical benefit have complicated the development of efficacious treatments for NAFLD.

\section{Patient and community perspectives}

5.1 People living with NAFLD can provide valuable insights into the design and implementation of interventions to safeguard and improve their health. Patients and patient organizations should be actively involved in developing policies and strategies to address NAFLD; however, few such groups currently address NAFLD.

5.2 Given that NAFLD is a largely invisible public health issue, high-profile patients can be especially useful in creating awareness and advocating for greater action on prevention and treatment.

5.3 Professional and patient organizations that address NCDs, including T2DM, obesity, heart disease and cancer, can play an important part in raising the profile of NAFLD, including by providing information to at-risk groups.

5.4 Stigma can be a major barrier when seeking to address health issues. Liver disease in general is commonly associated with unhealthy alcohol use, while NAFLD is associated with obesity. Both of these associations are with highly stigmatized conditions, and the implications of such stigma need to be acknowledged and addressed when developing prevention and treatment approaches for NAFLD.

\section{Policy strategies and a societal approach}

6.1 A national strategy for NAFLD is lacking in almost every country in the world, while NAFLD is explicitly mentioned in very few national strategies or clinical guidelines for related conditions such as obesity or diabetes. This fact highlights the extremely low priority the condition has in national health agendas, and the need for a concerted effort to shape and deliver a robust public health response.

6.2 Several highly prevalent NCDs share common risk factors - such as unhealthy diets, physical inactivity and unhealthy alcohol consumption - with NAFLD. Policies, fiscal measures and legislation could address many of these diseases in a coordinated, simultaneous way.

6.3 Addressing NAFLD will require collective action that spans diverse disciplines and sectors. Existing frameworks such as the United Nations Sustainable Development Goals (SDGs) can usefully inform and guide the development of multi-sectoral efforts to address the direct, underlying and cross-cutting causes of NAFLD.

\section{Leadership for the NAFLD public health agenda}

7.1 National and regional liver associations, in collaboration with governments and other stakeholders, have a leading role in responding to NAFLD, including in developing public health strategies and guidelines and in collaborating with other disease associations and organizations.

7.2 Multilateral organizations such as the WHO also have a key role in shaping and helping lead the response to NAFLD, firstly by recognizing the condition as a major health issue, and secondly by supporting nationally led efforts to deliver public health responses.

7.3 Global efforts to expand universal health coverage and ensure that health systems are people-centred provide a useful mechanism for holistically addressing NCDs, including not only NAFLD, but also associated diseases such as diabetes and obesity.

Grading system: $U$, denotes unanimous (100\%) agreement; A, 90-99\% agreement; B, 78-89\% agreement; and C, 67-77\% agreement. NAFLD, non-alcoholic fatty liver disease; NASH, non-alcoholic steatohepatitis; WHO, World Health Organization. 
Table 4 | Consensus recommendations for a NAFLD public health agenda

Number $\quad$ Recommendation
\begin{tabular}{|l} 
1. The human and economic burden \\
1 Investment is needed in research that will improve understanding of NAFLD epidemiology, \\
especially in under-studied population groups such as children, and people without overt \\
metabolic risk factors.
\end{tabular}
$\begin{aligned} & \text { In the absence of population-based and prospective longitudinal studies, alternate research } \\
& \text { methods should be considered, such as those employing electronic health records. }\end{aligned}$
$\begin{aligned} & \text { Investment cases should be developed for NAFLD at global, regional and local levels. To support } \\
& \text { these cases, toolkits should be prepared to provide guidance on obtaining the requisite economic } \\
& \text { data and communicating the findings to policy-makers, health-care funders and/or payers and } \\
& \text { other relevant stakeholders. }\end{aligned}$

\section{Awareness and education}

4 Professional societies and other relevant stakeholders, such as patient organizations, should collaborate on a transparent process to carefully reconsider the nomenclature of fatty liver diseases, with special attention to the benefits of and barriers to changing the name of 'non-alcoholic fatty liver disease'.

5 The liver health community should engage health communication experts to jointly develop effective strategies and practical tools to increase awareness in key audiences, including the media and policy-makers.

6 The terminology and concept of 'compensated advanced chronic liver disease' should be adopted, as it better reflects the continuum of advanced disease and the increased risk of decompensation than the current usage of fibrosis stages 3 and 4.

$7 \quad$ Professional bodies should develop simple knowledge products and educational courses targeting the liver and gastroenterology communities, primary care providers and specialists from other disciplines, as well as at-risk populations, the media and policy-makers. The courses should include medical school and continuing medical education activities.

\section{Defining and implementing models of care}

$8 \quad$ Health-care planners and providers should design and implement locally feasible NAFLD care pathways, utilizing available tests to efficiently determine a patient's care needs and link them to appropriate services.

$9 \quad$ Health-care providers - especially primary care providers, diabetes specialists and those caring for people living with obesity - should be equipped with the tools and knowledge needed to support the care of people living with NAFLD. At a minimum, providers should be able to identify which patients require referral to a liver specialist.

10 Multidisciplinary care models should form the basis for managing people living with NAFLD, especially those with advanced fibrosis.

11 Research should focus on developing more effective and more accurate non-invasive tests (NITs) for risk-stratifying patients - including children - in primary care, and for staging fibrosis and diagnosing NASH in secondary care.

12 Implementation research should be undertaken to better understand the barriers to uptake of currently available NITs.

13 Active case finding should be considered in population groups at high risk for advanced fibrosis. The specific target populations ought to be determined locally but should include people living with type 2 diabetes mellitus and central adiposity.

14 Implementation research is needed to identify the core elements of effective NAFLD care models in different health-care settings - including low-resource settings - and to provide generalizable findings that can inform the development of models of care in different contexts.

15 Preventing and treating childhood NAFLD should be a priority, both as a means of improving child health and as a way of reducing the burden of disease in later life.

\section{Treatment and care}

16 Research should focus on identifying interventions, including lifestyle treatments (for example, diet and physical activity regimens) and pharmacological treatments that can help people living with NAFLD and obesity to achieve and sustain a weight loss of at least $10 \%$.

17 Effective structured lifestyle treatment programmes should be made available to people living with NAFLD, especially those who are at high risk of advanced fibrosis and/or rapid fibrosis progression.

18 Currently accepted surrogate histological end points for conditional NASH drug approval should be standardized, with the goal of eventually replacing them with non-invasive diagnostic and surrogate end point biomarkers. 
Table 4 (cont.) | Consensus recommendations for a NAFLD public health agenda

\begin{tabular}{|c|c|c|}
\hline Number & Recommendation & Grade \\
\hline \multicolumn{3}{|c|}{ 5. Patient and community perspectives } \\
\hline 19 & $\begin{array}{l}\text { Medical associations and other stakeholders should support patient groups in meeting the needs } \\
\text { of people living with NAFLD. Where possible, NAFLD-specific groups should be formed. Patient } \\
\text { groups focused on related conditions - including diabetes and obesity - should be provided } \\
\text { with relevant information on NAFLD to share with their members. }\end{array}$ & A \\
\hline 20 & $\begin{array}{l}\text { Patient groups for liver disease and related non-communicable diseases (NCDs) should be } \\
\text { involved in the development of clinical practice guidelines for NAFLD. Medical associations } \\
\text { should also support these patient groups in developing relevant materials on NAFLD for their } \\
\text { members. }\end{array}$ & A \\
\hline \multicolumn{3}{|c|}{ 6. Policy strategies and a societal approach } \\
\hline 21 & $\begin{array}{l}\text { Efforts to detect, prevent and treat NAFLD should be integrated within a broader package } \\
\text { of cost-effective interventions that holistically address NCD risk factors, focusing specifically } \\
\text { on unhealthy diets, physical inactivity and unhealthy alcohol consumption. }\end{array}$ & A \\
\hline 22 & $\begin{array}{l}\text { Global health organizations (including the WHO) and national institutions should incorporate } \\
\text { NAFLD into their technical materials on NCDs and include NAFLD among their priority NCDs. }\end{array}$ & A \\
\hline 23 & $\begin{array}{l}\text { The WHO should dedicate a World Health Day }(7 \text { April) to liver health to highlight the global } \\
\text { prevalence of NAFLD and its significance for public health. }\end{array}$ & A \\
\hline 24 & $\begin{array}{l}\text { The NAFLD prevention agenda should include the creation of healthier, more equitable and } \\
\text { sustainable societies as one of its core goals. One way to do that should be to emphasize the SDG } \\
\text { targets that are relevant to preventing and treating NAFLD. }\end{array}$ & A \\
\hline \multicolumn{3}{|c|}{ 7. Leadership } \\
\hline 25 & $\begin{array}{l}\text { A global coalition of organizations and individuals should lead the development of a NAFLD } \\
\text { public health roadmap and support the global health community in following it. }\end{array}$ & A \\
\hline 26 & $\begin{array}{l}\text { Medical societies that provide care for any aspect of metabolic syndrome should formally } \\
\text { collaborate to address NAFLD, including by jointly developing guidelines, policy briefs and plans } \\
\text { of action. }\end{array}$ & A \\
\hline
\end{tabular}

Grading system: $\mathrm{U}$, denotes unanimous (100\%) agreement; A, 90-99\% agreement; B, 78-89\% agreement; and C, 67-77\% agreement. NAFLD, non-alcoholic fatty liver disease; NASH, non-alcoholic steatohepatitis; WHO, World Health Organization.

study settings, the race and ethnicities of the studied populations and the reference methods used to define NAFLD. A 2015 meta-analysis estimated the prevalence of NAFLD in children aged $1-19$ years at $7.6 \%(95 \%$ CI $5.5-10.3 \%$ ), rising to $34.2 \%$ (95\% CI $27.8-41.2 \%$ ) in studies conducted in paediatric obesity clinics ${ }^{39}$. NAFLD prevalence is generally higher in children living with obesity than in those without obesity ${ }^{39-44}$, but the extent of this relationship is likely to differ by population group ${ }^{45}$. NAFLD is also a public health problem in children and adolescents with normal weight ${ }^{44}$. Driven in part by rising obesity levels, the burden of childhood NAFLD has increased over the past three decades with an estimated annual change of $1.35 \%$ (95\% CI $1.16-1.54 \%)^{46}$.

Understanding the natural history, pathophysiology and phenotypes of childhood and adolescent NAFLD has advanced in the past two decades, including through articulation of clinically relevant subtypes of paediatric $\mathrm{NASH}^{47-49}$. NAFLD in children with T2DM has a unique pathological phenotype, which seems to be more aggressive than the adult form ${ }^{50}$. Further research is still needed to elucidate the pathophysiology, genetics, natural history and responses to treatment in paediatric NAFLD ${ }^{47}$ and therefore inform prevention and management approaches.

There are fewer data on the long-term impact of NAFLD in childhood than of NAFLD developed in later life. A Danish study estimated that for every 1 unit increase in BMI for-age $Z$-score between the ages of 7 and 13 years, the risk of cirrhosis increased by $16 \%{ }^{51}$. Another study in the same age group showed that a 1 unit increase in BMI increased the risk of liver cancer 30 years later by $20-30 \%{ }^{52}$. Weight gain in childhood or late adolescence is associated with a greater risk of NAFLD than weight gain in late adulthood ${ }^{20}$, although a high BMI in late adolescence increases the risk of severe liver disease in adulthood, independent of alcohol consumption ${ }^{53,54}$. A study of paediatric and young adult patients with biopsy-confirmed NAFLD in Sweden showed that, compared with matched controls, the patients with NAFLD had substantially higher rates of all-cause, cancer, liver and cardiometabolic-specific mortality ${ }^{55}$. More data on the long-term consequences of childhood NAFLD, including the life-time risk of developing cirrhosis, will help to inform strategies for prevention and management.

Quality of life. NAFLD research has started to explore the effect of the disease on affected populations using patient-reported outcome (PRO) data. PROs enable researchers and clinicians to look beyond clinical and histological outcomes to understand better the full impact of a condition. PROs capture health status from the perspective of the patient, from general quality of life (QoL) and HRQoL to work productivity, fatigue and satisfaction. Such information enables a comprehensive understanding of disease impact at the individual and 


\section{CONSENSUS STATEMENT}

\section{Box 1 | The NAFLD name debate}

Since the early 2000 s, several proposals have been made to change the name nonalcoholic fatty liver disease (NAFLD). The central arguments for change have been that the adjective 'non-alcoholic' is an unhelpful construction, and that other terms would better reflect the metabolic underpinnings of the disease's aetiology ${ }^{147}$. In the absence of widespread consensus, however, NAFLD has remained the commonly used nomenclature. In the past 2 years, the term 'metabolic dysfunction-associated fatty liver disease' (MAFLD) has gained traction as a possible replacement. An international group of experts from 22 countries reached consensus on the change to MAFLD ${ }^{148,149}$, and the proposed change was endorsed by regional liver associations in South America ${ }^{150}$ and the Asian Pacific ${ }^{115}$ as well as by experts in sub-Saharan Africa ${ }^{151}$ and the Middle East and North Africa ${ }^{152}$. However, other experts have expressed concerns about prematurely changing the name without fully considering its broad implications, from diagnostic criteria to trial end points, calling instead for regional liver societies to work together to reach consensus ${ }^{153,154}$.

societal levels. Several PRO tools have been developed and validated for use in people living with NAFLD ${ }^{56-58}$. Overall, QoL worsens with disease progression ${ }^{59}$. People living with NAFLD report worse QoL than those living without the disease, people living with NASH report worse QoL than those with NAFL ${ }^{60,61}$, and patients with cirrhotic NASH report worse HRQoL than patients with non-cirrhotic $\mathrm{NASH}^{2}$. The association between disease stage and HRQoL varies among countries and regions $^{3}$, highlighting the importance of local data. Research should aim to further our understanding of the outcomes most relevant to people living with NAFLD, so that policies and management strategies can be designed to minimize the effects of the disease on those affected.

Economic burden. In addition to the human burden, NAFLD also has wide-ranging economic implications for affected populations and societies at large ${ }^{3,4,6-8}$, including both direct medical expenses and indirect costs associated with consequences such as loss of work. Most economic costs associated with NAFLD are incurred in the latter stages of the disease $e^{4,7,8}$, providing a good rationale for funding prevention and early intervention efforts. Investment cases should be developed for NAFLD at global, regional and local levels. To support their development, toolkits should be prepared to provide guidance on obtaining the requisite economic data and communicating the findings to policy-makers, health-care funders and payers and other relevant stakeholders.

The vast human and economic impact of NAFLD provides a compelling imperative for action. More and better data on NAFLD, especially in under-studied populations such as children, are needed to advance our understanding of the impact of the disease and to shape health system and public health responses accordingly. Data disaggregated by disease stage, gender, age, ethnicity and geographical area will be critical. In the absence of population-based and longitudinal studies, alternative research methods should be explored. Electronic health records are one potentially valuable resource ${ }^{62}$. The latest efforts to standardize the administrative codes used to record exposures and outcomes for NAFLD will improve the feasibility of such research and facilitate comparisons between study populations ${ }^{63}$. As our understanding of the basic science and epidemiology of NAFLD grows, it will also be important to explore the effectiveness of different operational models on patient outcomes and resource utilization.

The lack of data on the human and economic burden of NAFLD not only inhibits our ability to deliver proportionate health system and public health responses, but to raise awareness of the disease and its consequences among key stakeholders, including policy-makers and at-risk groups. As we strive to better understand the epidemiology of NAFLD, the liver health community will also need to consider how to communicate these findings to different target audiences.

\section{Awareness, education and terminology \\ - Statements 2.1-2.3 \\ - Recommendations 4-7}

Despite being the most prevalent liver disease in history, NAFLD remains largely unknown outside hepatology and gastroenterology. Knowledge of NAFLD among general practitioners ${ }^{64}$ and non-liver health specialists is generally poor, with little sense of the scale of the challenge or the potential gravity of the disease ${ }^{65}$. Patients at higher risk of NAFLD, including people with T2DM and other metabolic risk factors, are also unaware of the disease, their susceptibility to developing it or how it interacts with other metabolic conditions ${ }^{66-68}$. There are limited data on NAFLD awareness amongst the general public, but what is available points to low levels of awareness $^{69}$.

Increasing awareness of NAFLD will require simple, effective messages and non-stigmatizing terminology that describe risk factors and potential consequences of the disease. Such messages need to be targeted to specific audiences, including health-care professionals especially hepatologists, gastroenterologists, primary care providers and diabetes specialists - policy-makers and the general public. Health communication experts and the media should be enlisted in developing awareness strategies and tools.

In addition, the liver health community needs to agree upon the terminology we use to describe the disease and its consequences. Compensated advanced chronic liver disease (cACLD) is a relatively new term for the early phases of severe chronic liver disease, covering severe fibrosis and compensated cirrhosis ${ }^{70}$. Adopting this term would improve clinical care and research, as cACLD better reflects the continuum of advanced disease and the increased risk of decompensation than the current use of fibrosis stages 3 and 4 (REFS ${ }^{70,71}$ ). Long-standing debates about the nomenclature used for fatty liver disease have also gained traction in the past few years, with 'metabolic dysfunction-associated fatty liver disease' (MAFLD) as a possible replacement for NAFLD (BOX 1). We urge the relevant organizations to engage in a thorough process to achieve consensus on the path forwards. The current lack of clarity risks fragmenting and confusing the liver health community, which would undoubtedly impede efforts to bring much needed attention and action to this critical public health issue. Beyond the clinical and scientific considerations, such a process should also address how a name change might facilitate efforts to increase 
awareness about the disease in an audience that is as wide as possible.

\section{Defining and implementing models of care \\ - Statements 3.1-3.12 \\ - Recommendations 8-15}

A model of care $(\mathrm{MoC})$ is a setting-specific framework that outlines how patients with a disease are managed along the care cascade. A comprehensive MoC outlines which services are to be provided, where they should be provided and by whom, and how they are to be integrated and coordinated within a health-care system $^{72}$. Clearly defined, context-specific MoCs will be important for managing the burden of NAFLD, and establishing such MoCs should be a key focus for health-care decision-makers and providers. Yet NAFLD MoCs have received little attention to date, with a review published in 2021 identifying only seven published examples of comprehensive MoCs, only one of which addresses children ${ }^{73}$.

The majority of patients with NAFLD can be managed in primary care. For patients with isolated steatosis or early-stage fibrosis in the primary care setting, management should focus on preventing disease progression and the development or exacerbation of metabolic comorbidities. Patients with advanced fibrosis might require a hepatologist or gastroenterologist to manage the hepatic component of the disease $\mathrm{e}^{74,75}$, whereas a smaller proportion will require tertiary care, such as for transplant surgery ${ }^{13,14}$.

As a multisystem, comorbid disease, people living with NAFLD will often benefit from multidisciplinary care, especially those with advanced fibrosis ${ }^{76}$. Establishment of multidisciplinary teams (MDTs) can be an effective way to manage the diverse clinical needs of people living with NAFLD ${ }^{76}$. There are several published examples of multidisciplinary secondary care clinics for NAFLD $^{77-81}$. Each provides a model of what is feasible and appropriate within a given health-care setting, with the composition and structure of the MDT and the services it provides varying accordingly.

Development of care pathways. The first step in an $\mathrm{MoC}$ is to identify each patient's needs, as determined by disease stage and presence of comorbidities, and to link them to appropriate services - a process known as risk stratification. However, diagnosing and staging NAFLD remains challenging, and diagnoses are often incidental to the identification of abnormal liver enzymes or of steatosis through imaging techniques, neither of which provides information on disease severity ${ }^{82}$.

A care pathway is a framework to support decisionmaking, including deciding when to refer a patient to specialist care. There are several published examples of care pathways for identifying advanced liver disease $\mathrm{e}^{80,81,83-87}$, and some evidence for the costeffectiveness of these approaches ${ }^{8-90}$. Yet formal pathways do not exist in many health-care settings, and non-invasive tests (NITs) are not routinely used in some settings where they might prove beneficial. Although the availability of specific NITs will vary, it is feasible to implement non-commercial blood-based tests in most primary and secondary care settings. Managing the burden of NAFLD requires developing locally appropriate care pathways and equipping health-care providers with the tools and knowledge to implement them. That is especially true for primary care providers, as many people living with NAFLD will first present in primary care, where the condition is widely under-diagnosed ${ }^{91}$. Another key setting is diabetes clinics, where the prevalence of advanced disease is higher than in the general population $^{18,24}$. Care pathways will also ensure that the necessary health-care infrastructure is in place when more effective pharmacological treatments become available. In the case of hepatitis $C$, such pathways were not adequately in place before all-oral direct-acting antiviral treatment became available, hampering efforts to link people with treatment ${ }^{72,92}$.

Care pathways for children living with NAFLD should also address the transition from paediatric to adult services to ensure continuity of care. These pathways need to recognize the differences in the clinical management of children and adults and the psychological factors associated with such a transition ${ }^{93}$.

Testing and screening for NAFLD. Fibrosis stage is a key indicator for long-term liver and non-liver health outcomes in patients with NAFLD ${ }^{94}$. Various NITs have been validated for detecting advanced fibrosis in clinical practice, ranging from blood-based scores to imaging techniques ${ }^{95}$. The performance of these NITs is strongly influenced by pretest probability. In primary care settings where the population prevalence of advanced disease is low, the negative predictive value of NITs for advanced fibrosis is generally high, whereas the positive predictive value is lower ${ }^{96,97}$. NITs can be especially effective at identifying advanced disease when used in sequential algorithms ${ }^{98-101}$. There is also some evidence that certain combinations of NITs can identify patients who have fibrosis stage 2 or greater with a high positive predictive value ${ }^{102}$. Although several NITs have been investigated for use in paediatric populations, none of them is currently validated for use in routine clinical practice. Initial screening in children generally relies on liver enzymes and ultrasonography, with a biopsy required to definitively diagnose and stage the disease. There is hope that NIT combinations might replace the need for biopsies in paediatric populations in the near future $^{103}$. The development of more efficient and effective NITs for risk-stratifying patients in primary care and diagnosing and staging NASH in secondary care remains a research priority.

There is broad consensus that certain factors, particularly T2DM and obesity, increase the risk of an individual developing NAFLD and of the disease progressing. However, guidance varies on the benefits and cost-effectiveness of active case finding in specific patient groups (BOX 2). Although appropriate targets for active case-finding should be determined with local epidemiology and resources in mind, the expert panel recommends that it include people living with T2DM and those with central adiposity. These approaches should be evaluated for their impact on patient outcomes and for cost-effectiveness. 


\section{CONSENSUS STATEMENT}

\section{Box 2 | Guidance on active case-finding in high-risk population groups}

Joint guidance from The European Association for the Study of the Liver (EASL), the European Association for the Study of Diabetes (EASD) and the European Association for the Study of Obesity (EASO) recommends screening for non-alcoholic fatty liver disease (NAFLD) in people with obesity, metabolic syndrome and in particular type 2 diabetes mellitus (T2DM) ${ }^{111}$. The Latin American Association for the Study of the Liver $(A L E H)^{112}$, the Asian Pacific Association for the Study of the Liver (APASL) ${ }^{115}$ and the Asia-Pacific Working Party on NAFLD ${ }^{155}$ all recommend considering screening in certain high-risk populations, including those with obesity and T2DM. The American Diabetes Association recommends screening for non-alcoholic steatohepatitis and advanced fibrosis in patients with elevated liver enzyme levels or hepatic steatosis on ultrasonography ${ }^{156}$. By contrast, the American Association for the Study of Liver Diseases (AASLD) does not recommend systematic screening in these groups, given the lack of cost-effectiveness data for such efforts ${ }^{113}$.

Primary care interventions. Access to high-quality primary care preventive interventions is critical to reducing the burden of NCDs ${ }^{104}$, yet there is little evidence for which primary care interventions will optimize patient outcomes for people living with NAFLD. However, the common risk factors for NAFLD, obesity, T2DM and CVD, including an unhealthy diet and physical inactivity ${ }^{105}$, argue for integrated chronic disease management approaches. Structured management programmes for other conditions, such as diabetes, can serve as a starting point for more integrated models ${ }^{106}$. In low-resource settings, the World Health Organization (WHO) package of essential NCD interventions for primary health care can be used as a basis for integrating NAFLD care into related disease areas, including diabetes management ${ }^{107}$. Technological innovation, such as health information exchanges and mHealth (mobile health) applications, can also help facilitate collaboration between patients and providers and the coordination of services within a health-care system by ensuring the timely and accurate flow of information ${ }^{108}$.

As the liver community leads efforts to improve the life of those with NAFLD, it should prioritize operational research that furthers our understanding of the effect of different MoCs on patient outcomes and of the cost-effectiveness of these approaches in different health-care settings. This research should also address the structural barriers that make coordination and collaboration within health-care systems a challenge and how to effectively engage across disciplines.

\section{Treatment and care}

\section{- Statements 4.1-4.4}

\section{- Recommendations 16-18}

The treatment and care of patients with NAFLD are highly dependent on their disease stage ${ }^{75}$. Interventions aimed at modifying lifestyle risk factors - namely weight, diet and physical activity - and at the management of comorbidities should be the cornerstone of treatment for all patients ${ }^{75,109,110}$. This priority is emphasized in the clinical management guidelines from regional liver associations ${ }^{111-115}$. In patients with more advanced disease, addressing components of metabolic syndrome, liver-related pharmacotherapy and management of cirrhosis-related complications are all important ${ }^{75,116}$. Even when effective pharmacological treatments for NAFLD become available, programmes aimed at modifying lifestyle risk factors should continue to be a core element of NAFLD disease management.

There is some evidence that such lifestyle interventions can prevent disease progression and, in some cases, reverse fibrosis ${ }^{117,118}$. In persons affected by overweight and obesity, NAFLD lifestyle interventions aim to achieve and sustain a weight loss of around $10 \%$, which is associated with the improvement of liver enzyme levels and histological findings ${ }^{111-113}$. Behavioural change approaches are most effective when incorporated into a comprehensive, long-term lifestyle modification programme ${ }^{119}$. Dietary guidance for people living with NAFLD generally centres on the reduction of saturated fats, sugar-sweetened beverages, refined carbohydrates and red and processed meats ${ }^{120,121}$. The Mediterranean diet and the Dietary Approaches to Stop Hypertension (DASH) have proven beneficial in some patients by improving liver status, in particular hepatic insulin sensitivity and lipid profile ${ }^{120,122}$. Several forms of physical activity - aerobic, resistance or high-intensity intervals - seem to have a beneficial effect on liver fat ${ }^{118,123}$. Even in the absence of weight loss, exercise can result in a $20-30 \%$ reduction in intrahepatic lipid levels ${ }^{117,118}$. It is important that diet and exercise programmes are tailored to the patient's needs and preferences to support long-term adherence. Further research is needed to identify the interventions, whether lifestyle or pharmacological interventions, that are most effective in helping people living with NAFLD and obesity to achieve and sustain a weight loss of at least $5-10 \%$ of initial body weight. Research is also needed to determine how best to implement lifestyle interventions, including research on how different operational models influence long-term adherence and patient outcomes, and on the cost-effectiveness of different approaches. Collaboration between disciplines, including basic, behavioural and clinical sciences and operations researchers, among others, will help to advance our understanding in this area in the years to come.

Making effective structured lifestyle treatment programmes available to people with NAFLD, especially those who are at high risk of advanced fibrosis and/or rapid fibrosis progression, such as people living with NAFLD, obesity and T2DM, should be a priority of the liver health community. Both public and private funders will have a key part to play in ensuring financial support for such services. As a first step, NAFLD needs to be adequately incorporated into relevant national health-care policies and guidelines, something that is currently lacking in most countries ${ }^{124}$.

Although there are currently no pharmacological treatments specifically approved for NAFLD, clinical trials are exploring numerous drug candidates targeting energy intake, energy disposal, lipotoxic liver injury, inflammation and fibrosis ${ }^{125}$. The invasive nature of liver biopsy, the inherent variability of histological findings and the lack of an alternative validated surrogate for long-term clinical benefit have complicated the development of efficacious treatments. As the field moves forwards, it would be helpful to standardize the surrogate histological end points that are currently accepted 
for conditional NASH drug approval, with the goal of eventually replacing them with non-invasive diagnostic and surrogate end point biomarkers.

\section{Patient and community perspectives \\ - Statements 5.1-5.4 \\ - Recommendations 19-20}

People with NAFLD can provide valuable insights into the design and delivery of interventions to safeguard and improve their health. Actively engaging people with lived experiences, especially disproportionately affected communities such as certain minority ethnic groups, and considering their perspectives will help ensure that interventions are patient-centred, improving treatment adherence and outcomes ${ }^{126,127}$. Developing education materials that are specific to population groups and reflect diverse backgrounds will also improve outreach and engagement efforts.

Patient groups have a critical role in advocating for greater attention to under-served health issues and as a source of information for the affected population (BOX 3). In thinking about developing a patient community for NAFLD, the history of the viral hepatitis movement is instructive. Patient organizations had a pivotal role in that movement, notably through driving World Health Assembly Resolution 67.6, which was adopted in May 2014 (REF. $\left.{ }^{128}\right)$. The resolution framed viral hepatitis as a major public health problem that required comprehensive global and national action. The World Hepatitis Alliance was key to this success, providing a platform that united diverse organizations around a common vision. Although there is no global patient alliance for NAFLD, there are platforms within the wider NCD community, such as the NCD Alliance, that liver health organizations can engage with to increase awareness of NAFLD. These efforts can complement the ongoing work of organizations within the liver health community.

One challenge in engaging people living with NAFLD is the fear of stigma. People commonly connect liver disease with unhealthy alcohol use, while NAFLD is often connected with obesity and its associated stereotypes (such as laziness); both of these conditions are highly stigmatized ${ }^{28,129-131}$. People living with multiple chronic conditions can also experience multiple interacting

\section{Box $3 \mid$ The importance of patient groups}

Patient organizations are often a primary source of information for affected populations whilst also providing a platform through which patients can engage in developing policies and strategies, including clinical practice guidelines. However, globally, few non-alcoholic fatty liver disease (NAFLD)-specific patient groups exist and there is no truly global platform or coordination mechanism to support local patient organizations. The liver community should further mobilize financial and technical support to help form such groups. We suggest that professional and patient organizations that address associated conditions such as obesity, type 2 diabetes mellitus, heart disease and cancer can also have an important role in disseminating information to people in high-risk groups. Medical associations will need to lead efforts to develop information tools and support the dissemination of these amongst key target groups. As the meaningful engagement of people with lived experiences is becoming more mainstream in the non-communicable disease field, including within the World Health Organization $(\mathrm{WHO})^{157}$, the liver health community should take the opportunity to prioritize this issue into the NAFLD agenda. forms of stigma ${ }^{132}$. The implications of stigma need to be acknowledged and addressed when developing prevention and treatment approaches for NAFLD. High-profile individuals living with NAFLD could have a great impact in creating awareness and advocating for prevention and treatment efforts, as well as in reducing any stigma associated with the condition.

\section{Policy strategies and a societal approach \\ - Statements 6.1-6.3 \\ - Recommendations 21-24}

Despite being a highly prevalent liver disease, little attention has been paid to the policies and strategies needed to prevent, manage and treat NAFLD. A review of 29 European countries examining the existence of policies for NAFLD found large variations in national responses, and none of the countries was prepared to address the challenge ${ }^{124,133}$. A global study of 102 countries painted a similar picture, highlighting an overall lack of attention to NAFLD in national health agendas; not a single country reported having a written NAFLD strategy. Even in national strategies and clinical guidelines for related conditions such as obesity or T2DM, NAFLD is seldom mentioned. These findings highlight the extremely low priority the condition has in both disease-specific and national health agendas, and the need for a concerted effort to shape and deliver a robust public health response ${ }^{134}$. Fortunately, there is some cause for optimism, with efforts such as the US NASH action plan by the NASH Council and the Global Liver Institute published in December 2020 that provides a model for others to consider ${ }^{135}$.

At a health system level, chronic disease management is driving the reorientation of health systems away from siloed disease-centred models to multidisciplinary patient-centred care ${ }^{136,137}$. The liver community can, in collaboration with other actors working with metabolic disease management, help lead this process in the coming years for the benefit of not only patients with liver disease but of all people living with NCDs.

At both a public health level and a clinical management level, there is substantial overlap in the measures required to address NAFLD and the other major NCDs. Common risk factors, such as unhealthy diets, physical inactivity and unhealthy alcohol consumption, provide an opportunity for collaborative approaches to improve public health. Policies, fiscal measures and legislation that address common risk factors for NCDs in a coordinated, synergistic way have the potential to create a lasting impact. Yet despite the common approaches needed to address NAFLD and other NCDs, NAFLD is not mentioned by name in the majority of key global or national NCD strategies; most notably, it is absent from the WHO Action Plan on the Prevention and Control of $\mathrm{NCDs}^{25}$. Liver health organizations must engage more effectively with $\mathrm{WHO}$ and other national and international organizations to ensure that measures to prevent and treat NAFLD are fully integrated into a broader package of cost-effective interventions that address NCD risk factors holistically. Each year World Health Day is marked on 7 April and the theme changes each year, providing the opportunity to shine a spotlight 
on key issues. Dedicating a World Health Day to liver health would provide a platform for NAFLD advocacy and awareness-raising within the global health field and beyond. Such a day would complement existing advocacy and awareness efforts, including International NASH Day, which is marked on 12 June each year.

Complex health issues also require us to rethink systems and go beyond the immediate determinants of a disease to consider the underlying influences and root causes, as well as the multidisciplinary and multisectoral responses needed to address these ${ }^{138}$. The liver health field must look beyond the health sector as it seeks to address the challenges of NAFLD. It can take lessons from other fields such as obesity, in which thinking has evolved during the past two decades beyond a focus on individual-level factors underlying energy imbalances to a consideration of the biological, social, environmental and policy drivers of health behaviours and outcomes $^{139}$ and a systems approach to the ways these drivers interact ${ }^{140,141}$. Such an approach calls for coordinated actions from all stakeholders to improve policies and practices spanning multiple sectors and to shift social norms on health ${ }^{142,143}$.

The NAFLD prevention agenda should therefore include the creation of healthier, more equitable and sustainable societies as part of its vision. Existing frameworks such as the United Nations Sustainable Development Goals (SDGs) can usefully inform and guide the development of multisectoral efforts to address NAFLD. A recently developed SDG framework for NAFLD (available as a preprint) aims to help conceptualize thinking about the design and delivery of such responses ${ }^{144}$. As a first step, this framework can be used as a strategic advocacy tool to build the case for closer collaboration within and among sectors.

\section{Leadership for the NAFLD public health agenda \\ - Statements 7.1-7.3 \\ - Recommendations 25-26}

To move the NAFLD public health agenda forwards, national, regional and international liver associations, in collaboration with governments and other stakeholders, will need to lead the way. Multilateral organizations such as $\mathrm{WHO}$ also have a key role in shaping and delivering responses to NAFLD, first by recognizing the condition as a public health issue worthy of attention, and second by supporting national public health responses.

Several existing policy levers and movements can support the development of such responses too. For instance, global efforts to expand universal health coverage and ensure that health systems are people-centred provide useful mechanisms for addressing NCDs holistically. In addition, medical societies whose members provide health care for aspects of metabolic syndrome are well positioned to help lead this change. NAFLD should also be incorporated, whenever appropriate, in the development of joint plans of action, guidelines, policy briefs and educational tools, and these efforts should be adequately resourced. Finally, a global coalition of NAFLD stakeholders, both organizations and individuals, should lead the development of a NAFLD public health roadmap and advocate for its adoption by the global health community. This coalition should actively engage with those outside the liver health space by growing and nurturing a broad network of individuals and organizations with a common vision and goals.

\section{Study strengths and limitations}

Although the Delphi method is the right approach for a consensus-building initiative, it is not without challenges and limitations ${ }^{145,146}$. We employed purposive sampling to select the members of the initial core group and then used core group member recommendations to generate a larger, more diverse expert panel. In doing so, we sought to mitigate concerns about the inherent bias in the purposive sampling of a relatively small group with more broad-based snowball sampling, which resulted in a diverse expert panel of 218 members from 91 countries/territories and all six WHO regions. The variety of backgrounds represented on the panel - including academia, civil society, government, private sector, research, clinical practice and advocacy - strengthened the validity of the consensus statements and recommendations. That said, we understand that conducting the study in English might have limited the composition of the expert panel and therefore the findings.

Delphi studies often involve a combination of in-person convenings for in-depth deliberation and survey rounds for voting. However, in light of the geographical spread of panel members and COVID-19 travel restrictions, we employed alternative modes of group discourse. The core group was convened virtually at two points in the process, while panel members were able to provide written comments on the draft recommendations and the three survey rounds. Although we received and incorporated a large volume of open-ended comments across all four data collection components, we acknowledge that this approach might not have resulted in the same outcomes as those that would have emerged from real-time discussion and resolution of complicated or contentious issues. Conversely, this method gave panel members multiple opportunities to provide open-ended comments in a space without any dominant voices whose presence sometimes inhibits the expression of minority viewpoints during in-person convenings. The combination of in-person feedback (from core group members) and written feedback (from the entire expert panel) might therefore have resulted in more comprehensive contributions overall.

The increasing levels of agreement with the consensus statements across all three survey rounds, together with the high levels of participation ( $88 \%$ in R1 and R2; $85 \%$ in R3; 79\% in the online meeting), strengthen our confidence in the rigor of the method used and the resultant findings. Expert panel members' ability to include detailed comments on each of the draft statements enabled us to improve them, as reflected in the increasing mean level of agreement with the statements in successive rounds, from $80.3 \%$ in $\mathrm{R} 1$ to $98.5 \%$ in $\mathrm{R} 3$. Moreover, the endorsement of the final consensus statements and recommendations presented in TABLES 3 and 4 by 110 organizations in 59 countries/territories (Supplementary Table 8) at the time of publication further testifies to their global relevance. 


\section{Conclusions}

NAFLD is a highly prevalent disease that poses a major challenge to global public health. In this Consensus Statement, a diverse international group of experts developed and endorsed a set of consensus statements and recommendations that provide needed guidance for the creation and implementation of health system and public health responses that will rise to this challenge. The public health approach that informed the consensus-building process helped ensure the relevance of these statements and recommendations for a broad group of stakeholders, from researchers and health-care providers to policy-makers and funders. It is now up to the liver health community to lead the development of a roadmap to translate these statements and recommendations into global vision and action.

Published online 27 October 2021
1. Golabi, P. et al. Non-alcoholic fatty liver disease (NAFLD) is associated with impairment of health related quality of life (HROOL). Health Qual. Life Outcomes 14, 18 (2016).

2. McSweeney, L. et al. Health-related quality of life and patient-reported outcome measures in NASH-related cirrhosis. JHEP Rep. 2, 100099 (2020).

3. O'Hara, J. et al. Cost of non-alcoholic steatohepatitis in Europe and the USA: the GAIN study. JHEP Rep. 2, 100142 (2020).

4. Schattenberg, J. M. et al. Disease burden and economic impact of diagnosed non-alcoholic steatohepatitis (NASH) in five European countries in 2018: a cost-of-illness analysis. Liver Int. 41, 1227-1242 (2021)

5. Younossi, Z. M. et al. The association of histologic and noninvasive tests with adverse clinical and patient-reported outcomes in patients with advanced fibrosis due to nonalcoholic steatohepatitis. Gastroenterology https://doi.org/10.1053/ j.gastro.2020.12.003 (2020).

6. Younossi, Z. M. et al. The economic and clinical burden of nonalcoholic fatty liver disease in the United States and Europe. Hepatology 64, 1577-1586 (2016).

7. Hagström, H. et al. Health care costs of patients with biopsy-confirmed nonalcoholic fatty liver disease are nearly twice those of matched controls. Clin. Gastroenterol. Hepatol. 18, 1592-1599.e8 (2020).

8. Gordon, S. C., Fraysse, J., Li, S., Ozbay, A. B. \& Wong, R. J. Disease severity is associated with higher healthcare utilization in nonalcoholic steatohepatitis Medicare patients. Am. J. Gastroenterol. 115, 562-574 (2020)

9. Bertot, L. C. \& Adams, L. A. The natural course of non-alcoholic fatty liver disease. Int. J. Mol. Sci. 17 774 (2016).

10. Ekstedt, M., Nasr, P. \& Kechagias, S. Natural history of NAFLD/NASH. Curr. Hepatol. Rep. 16, 391-397 (2017).

11. Araújo, A. R., Rosso, N., Bedogni, G., Tiribelli, C. \& Bellentani, S. Global epidemiology of non-alcoholic fatty liver disease/non-alcoholic steatohepatitis: what we need in the future. Liver Int. 38, 47-51 (2018).

12. Kanwal, F. et al. Risk of hepatocellular cancer in patients with non-alcoholic fatty liver disease. Gastroenterology 155, 1828-1837.e2 (2018).

13. Haldar, D. et al. Outcomes of liver transplantation for non-alcoholic steatohepatitis: a European Liver Transplant Registry study. J. Hepatol. 71, 313-322 (2019).

14. Terrault, N. A. \& Pageaux, G.-P. A changing landscape of liver transplantation: king HCV is dethroned, ALD and NAFLD take over! J. Hepatol. 69, 767-768 (2018).

15. Global Burden of Disease Cancer Collaboration et al. Global, regional, and national cancer incidence, mortality, years of life lost, years lived with disability, and disability-adjusted life-years for 29 cancer groups, 1990 to 2017: a systematic analysis for the Global Burden of Disease Study. JAMA Oncol. 5 1749-1768 (2019).

16. Adams, L. A., Anstee, Q. M., Tilg, H. \& Targher, G. Non-alcoholic fatty liver disease and its relationship with cardiovascular disease and other extrahepatic diseases. Gut 66, 1138-1153 (2017).

17. Anstee, Q. M., Targher, G. \& Day, C. P. Progression of NAFLD to diabetes mellitus, cardiovascular disease or cirrhosis. Nat. Rev. Gastroenterol. Hepatol. 10 330-344 (2013)

18. Byrne, C. D. \& Targher, G. NAFLD: a multisystem disease. J. Hepatol. 62, S47-S64 (2015).

19. Albhaisi, S., Chowdhury, A. \& Sanyal, A. J. Non-alcoholic fatty liver disease in lean individuals. JHEP Rep.: Innov. Hepatol. 1, 329-341 (2019).
20. Younossi, Z. et al. Global burden of NAFLD and NASH: trends, predictions, risk factors and prevention. Nat. Rev. Gastroenterol. Hepatol. 15, 11-20 (2018).

21. Vilar-Gomez, E. et al. Fibrosis severity as a determinant of cause-specific mortality in patients with advanced nonalcoholic fatty liver disease: a multi-national cohort study. Gastroenterology 155 , 443-457.e17 (2018)

22. Angulo, P. et al. Liver fibrosis, but no other histologic features, is asssociated with long-term outcomes of patients with nonalcoholic fatty liver disease. Gastroenterology 149, 389-397.e10 (2015).

23. Haflidadottir, S. et al. Long-term follow-up and liver-related death rate in patients with non-alcoholic and alcoholic related fatty liver disease. BMC Gastroenterol. 14, 166 (2014).

24. Park, S. K., Seo, M. H., Shin, H. C. \& Ryoo, J. H. Clinical availability of nonalcoholic fatty liver disease as an early predictor of type 2 diabetes mellitus in Korean men: 5-year prospective cohort study. Hepatology 57, 1378-1383 (2013).

25. World Health Organization. Global action plan for the prevention and control of noncommunicable diseases 2013-2020. WHO https://apps.who.int//iris/bitstream/ handle/10665/94384/9789241506236_eng.pdf (2013).

26. Bennett, J. E. et al. NCD countdown 2030: worldwide trends in non-communicable disease mortality and progress towards Sustainable Development Goal target 3.4. Lancet 392,1072-1088 (2018).

27. Rotondi, A. \& Gustafson, D. in Gazing into the Oracle: The Delphi Method and its Application to Social Policy and Public Health (eds Adler, M. \& Ziglio, E.) 34-55 (Jessica Kingsley, 1996).

28. Rubino, F. et al. Joint international consensus statement for ending stigma of obesity. Nat. Med. 26, 485-497 (2020)

29. Lazarus, J. V. et al. Consensus statement on the role of health systems in advancing the long-term well-being of people living with HIV. Nat. Commun. 12, 4450 (2021).

30. Vos, T. et al. Global burden of 369 diseases and injuries in 204 countries and territories, 1990-2019: a systematic analysis for the Global Burden of Disease Study 2019. Lancet 396, 1204-1222 (2020).

31. Younossi, Z. M. et al. Global epidemiology of nonalcoholic fatty liver disease-meta-analytic assessment of prevalence, incidence, and outcomes. Hepatology 64, 73-84 (2016).

32. Estes, C. et al. Modeling NAFLD disease burden in China, France, Germany, Italy, Japan, Spain, United Kingdom, and United States for the period 2016-2030. J. Hepatol. 69, 896-904 (2018).

33. Estes, C., Razavi, H., Loomba, R., Younossi, Z. \& Sanyal, A. J. Modeling the epidemic of nonalcoholic fatty liver disease demonstrates an exponential increase in burden of disease. Hepatology 67 , 123-133 (2018)

34. Younossi, Z. et al. Global perspectives on nonalcoholic fatty liver disease and nonalcoholic steatohepatitis. Hepatology 69, 2672-2682 (2019).

35. Ye, Q. et al. Global prevalence, incidence, and outcomes of non-obese or lean non-alcoholic fatty liver disease: a systematic review and meta-analysis. Lancet Gastroenterol. Hepatol. 5, 739-752 (2020).

36. Bjorkstrom, K., Stal, P., Hultcrantz, R. \& Hagstrom, H. Histologic scores for fat and fibrosis associate with development of type 2 diabetes in patients with nonalcoholic fatty liver disease. Clin. Gastroenterol. Hepatol. 15, 1461-1468 (2017).

37. Musso, G., Gambino, R., Cassader, M. \& Pagano, G Meta-analysis: natural history of non-alcoholic fatty liver disease (NAFLD) and diagnostic accuracy of non-invasive tests for liver disease severity. Ann. Med. 43, 617-649 (2011).
38. Sepanlou, S. G et al. The global, regional, and national burden of cirrhosis by cause in 195 countries and territories, 1990-2017: a systematic analysis for the Global Burden of Disease Study 2017. Lancet Gastroenterol. Hepatol. 5, 245-266 (2020).

39. Anderson, E. L. et al. The prevalence of non-alcoholic fatty liver disease in children and adolescents: a systematic review and meta-analysis. PLOS ONE 10, e0140908 (2015).

40. Jimenez-Rivera, C et al. Prevalence and risk factors for non-alcoholic fatty liver in children and youth with obesity. BMC Pediatr. 17, 113 (2017).

41. Xanthakos, S. A. et al. High prevalence of nonalcoholic fatty liver disease in adolescents undergoing bariatric surgery. Gastroenterology 149, 623-634.e8 (2015).

42. Yu, E. L. et al. Prevalence of nonalcoholic fatty liver disease in children with obesity. J. Pediatr. 207, 64-70 (2019)

43. Schwimmer, J. B. et al. Prevalence of fatty liver in children and adolescents. Pediatrics 118, 1388-1393 (2006).

44. Yüksel, F. et al. Fatty liver disease in an autopsy series of children and adolescents. Hippokratia 16, 61-65 (2012).

45. Rocha, R. et al. Nonalcoholic fatty liver disease in asymptomatic Brazilian adolescents. World J. Gastroenterol. 15, 473-477 (2009).

46. Zhang, $X$. et al. Increasing prevalence of NAFLD/NASH among children, adolescents and young adults from 1990 to 2017: a population-based observational study. BMJ Open 11, e042843 (2021).

47. Schwimmer, J. B. et al. Histopathology of pediatric nonalcoholic fatty liver disease. Hepatology $\mathbf{4 2}$, 641-649 (2005).

48. Nobili, V. et al. NAFLD in children: new genes, new diagnostic modalities and new drugs. Nat. Rev. Gastroenterol. Hepatol. 16, 517-530 (2019).

49. Vos, M. B. et al. Factors to consider in development of drugs for pediatric nonalcoholic fatty liver disease. Gastroenterology 157, 1448-1456.e1 (2019).

50. Nadeau, K. J. et al. Youth-onset type 2 diabetes consensus report: current status, challenges, and priorities. Diabetes Care 39, 1635-1642 (2016).

51. Zimmermann, E. et al. Body mass index in s chool-aged children and the risk of routinely diagnosed non-alcoholic fatty liver disease in adulthood: a prospective study based on the Copenhagen School Health Records Register. BMJ Open 5, e006998 (2015).

52. Berentzen, T. L., Gamborg, M., Holst, C., Sørensen, T. I. \& Baker, J. L. Body mass index in childhood and adult risk of primary liver cancer. J. Hepatol. 60, 325-330 (2014).

53. Hagström, H., Stål, P., Hultcrantz, R., Hemmingsson, T. $\&$ Andreasson, A. Overweight in late adolescence predicts development of severe liver disease later in life: a 39years follow-up study. J. Hepatol. 65 , 363-368 (2016)

54. Hagström, H., Tynelius, P. \& Rasmussen, F. High $\mathrm{BMI}$ in late adolescence predicts future severe liver disease and hepatocellular carcinoma: a national, population-based cohort study in 1.2 million men. Gut 67, 1536-1542 (2018)

55. Simon, T. G. et al. Non-alcoholic fatty liver disease in children and young adults is associated with increased long-term mortality. J. Hepatol. https://doi.org/ 10.1016/j.jhep.2021.06.034 (2021).

56. Younossi, Z. M. et al. A disease-specific quality of life instrument for non-alcoholic fatty liver disease and non-alcoholic steatohepatitis: CLDQ-NAFLD. Liver Int. 37, 1209-1218 (2017).

57. Doward, L. C. et al. Development of a patient-reported outcome measure for non-alcoholic steatohepatitis (NASH-CHECK): results of a qualitative study. Patient 14, 533-543 (2021). 
58. Twiss, J. et al. Development of a new patient-reported outcome measure for non-alcoholic steatohepatitis: NASH-CHECK [abstract PGI39]. Value Health 20. A638 (2017)

59. Huber, Y. et al. Health-related quality of life in nonalcoholic fatty liver disease associates with hepatic inflammation. Clin Gastroenterol Hepatol. 17, 2085-2092.e1 (2019).

60. David, K. et al. Quality of life in adults with nonalcoholic fatty liver disease: baseline data from the Nonalcoholic Ateatohepatitis Clinical Research Network. Hepatology 49, 1904-1912 (2009).

61. Kennedy-Martin, T., Bae, J. P., Paczkowski, R. \& Freeman, E. Health-related quality of life burden of nonalcoholic steatohepatitis: a robust pragmatic literature review. J. Patient Rep. Outcomes 2, 28 (2017).

62. Casey, J. A., Schwartz, B. S., Stewart, W. F. \& Adler, N. E. Using electronic health records for population health research: a review of methods and applications. Annu. Rev. Public Health 37, 61-81 (2016).

63. Hagström, $\mathrm{H}$. et al. Administrative coding in electronic health care record-based research of NAFLD: an expert panel consensus statement. Hepatology https://doi.org/10.1002/hep.31726 (2021).

64. Sanyal, A. J. Putting non-alcoholic fatty liver disease on the radar for primary care physicians: how well are we doing? BMC Med. 16, 148 (2018).

65. Bergqvist, C. J. et al. Awareness and opinions of non-alcoholic fatty liver disease by hospital specialists. Intern. Med. J. 43, 247-253 (2013).

66. Alemany-Pagès, M. et al. Insights from qualitative research on NAFLD awareness with a cohort of T2DM patients: time to go public with insulin resistance? BMC Public Health 20, 1142-1142 (2020).

67. Wieland, A. C. et al. Low awareness of nonalcoholic fatty liver disease among patients at high metabolic risk. J. Clin. Gastroenterol. 49, e6-e10 (2015).

68. Goh, G. B. B. et al. Perceptions of non-alcoholic fatty liver disease - an Asian community-based study. Gastroenterol. Rep. 4, 131-135 (2016).

69. Ghevariya, V. et al. Knowing what's out there awareness of non-alcoholic fatty liver disease. Front. Med. 1, 4 (2014).

70. Augustin, S., Pons, M., Santos, B., Ventura, M. \& Genescà, J. in Portal Hypertension VI: Proceedings of the Sixth Baveno Consensus Workshop: Stratifying Risk and Individualizing Care (ed. de Franchis, R.) 39-49 (Springer, 2016)

71. de Franchis, R. Expanding consensus in portal hypertension: report of the Baveno VI Consensus Workshop: Stratifying Risk and Individualizing Care for Portal Hypertension. J. Hepatol. 63 , 743-752 (2015)

72. Lazarus, J. V. et al. We know DAAs work, so now what? Simplifying models of care to enhance the hepatitis C cascade. J. Intern. Med. 286, 503-525 (2019).

73. Lazarus, J. V. et al. Defining comprehensive models of care for NAFLD. Nat. Rev. Gastroenterol. Hepatol. https://doi.org/10.1038/s41575-021-00477-7 (2021).

74. Grgurevic, I. et al. Natural history of nonalcoholic fatty liver disease: implications for clinical practice and an individualized approach. Can. J. Gastroenterol. Hepatol. 2020, 9181368 (2020).

75. Dyson, J. K., Anstee, Q. M. \& McPherson, S. Non-alcoholic fatty liver disease: a practical approach to treatment. Frontline Gastroenterol. 5, 277-286 (2014).

76. Ruissen, M. M., Mak, A. L., Beuers, U., Tushuizen, M. E. \& Holleboom, A. G. Non-alcoholic fatty liver disease: a multidisciplinary approach towards a cardiometabolic liver disease. Eur. J. Endocrinol. 183, R57-R73 (2020).

77. Ahmed, M. H., Woodward, C. \& Mital, D. Metabolic clinic for individuals with HIV/AIDS: a commitment and vision to the future of HIV services. Cardiovasc. Endocrinol. 6, 109-112 (2017).

78. Armstrong, M. J. et al. Severe asymptomatic non-alcoholic fatty liver disease in routine diabetes care; a multi-disciplinary team approach to diagnosis and management. OJM 107, 33-41 (2014).

79. DeVore, S. et al. A multidisciplinary clinical program is effective in stabilizing BMI and reducing transaminase levels in pediatric patients with NAFLD. J. Pediatr. Gastroenterol. Nutr. 57, 119-123 (2013).

80. Moolla, A. et al. A multidisciplinary approach to the management of NAFLD is associated with improvement in markers of liver and cardio-metabolic health. Frontline Gastroenterol. 10, 337-346 (2019).

81. Neilson, L. J. et al. Implementation of a care bundle improves the management of patients with non-alcoholic fatty liver disease. Frontline Gastroenterol. https://doi.org/10.1136/ flgastro-2020-101480 (2021).

82. Dyson, J. K., Anstee, O. M. \& McPherson, S Non-alcoholic fatty liver disease: a practical approach to diagnosis and staging. Front. Gastroenterol. 5 211-218 (2014).

83. Chalmers, J. et al. The development and implementation of a commissioned pathway for the identification and stratification of liver disease in the community. Front. Gastroenterol. 11, 86-92 (2020).

84. Dillon, J. F. et al. Intelligent liver function testing (iLFT): a trial of automated diagnosis and staging of liver disease in primary care. J. Hepatol. 71, 699-706 (2019).

85. Hudson, M. \& McPherson, S, on behalf of the North East $\&$ North Cumbria Hepatology Network. Liver Network: Guidelines for the Management of Adults with Asymptomatic Liver Function Abnormalities (Northern England Clinical Strategic Networks, 2020).

86. Shaheen, A. A. et al. Risk stratification of patients with nonalcoholic fatty liver disease using a case identification pathway in primary care: a cross-sectional study. CMAJ Open 8, E370-E376 (2020).

87. Srivastava, A. et al. Prospective evaluation of a primary care referral pathway for patients with non-alcoholic fatty liver disease. J. Hepatol. 71 371-378 (2019).

88. Crossan, C. et al. Referral pathways for patients with NAFLD based on non-invasive fibrosis tests: diagnostic accuracy and cost analysis. Liver Int. 39, 2052-2060 (2019).

89. Srivastava, A. et al. Cost-comparison analysis of FIB-4 ELF and fibroscan in community pathways for non-alcoholic fatty liver disease. BMC Gastroenterol. 19, 122 (2019).

90. Tanajewski, L. et al. Economic evaluation of a community-based diagnostic pathway to stratify adults for non-alcoholic fatty liver disease: a Markov mode informed by a feasibility study. BMJ Open 7, e015659 (2017).

91. Alexander, M. et al. Real-world data reveal a diagnostic gap in non-alcoholic fatty liver disease. BMC Med. 16, 130 (2018).

92. Cooke, G. S. et al. Accelerating the elimination of viral hepatitis: a Lancet Gastroenterology \& Hepatology Commission. Lancet Gastroenterol. Hepatol. 4 135-184 (2019).

93. Mencin, A. A., Loomba, R. \& Lavine, J. E. Caring for children with NAFLD and navigating their care into adulthood. Nat. Rev. Gastroenterol. Hepatol. 12, 617-628 (2015)

94. Taylor, R. S. et al. Association between fibrosis stage and outcomes of patients with nonalcoholic fatty liver disease: a systematic review and meta-analysis. Gastroenterology 158, 1611-1625. e12 (2020).

95. Castera, L., Friedrich-Rust, M. \& Loomba, R Noninvasive assessment of liver disease in patients with nonalcoholic fatty liver disease. Gastroenterology 156, 1264-1281.e4 (2019).

96. Vali, Y et al. Enhanced liver fibrosis test for the non-invasive diagnosis of fibrosis in patients with NAFLD: a systematic review and meta-analysis. J. Hepatol. 73, 252-262 (2020).

97. McPherson, S. et al. Age as a confounding factor for the accurate non-invasive diagnosis of advanced NAFLD fibrosis. Am. J. Gastroenterol. 112, 740-751 (2017).

98. Anstee, Q. M. et al. Noninvasive tests accurately identify advanced fibrosis due to NASH: baseline data from the STELLAR trials. Hepatology 70, 1521-1530 (2019).

99. Augustin, S. et al. Identification of patients with advanced fibrosis due to nonalcoholic fatty liver disease: considerations for best practice. J. Gastrointestin Liver Dis. 29, 235-245 (2020)

100. Castera, L. Diagnosis of non-alcoholic fatty liver disease/non-alcoholic steatohepatitis: non-invasive tests are enough. Liver Int. 38(Suppl 1), 67-70 (2018).

101. Majumdar, A., Campos, S., Gurusamy, K., Pinzani, M. $\&$ Tsochatzis, E. A. Defining the minimum acceptable diagnostic accuracy of noninvasive fibrosis testing in cirrhosis: a decision analytic modeling study. Hepatology 71, 627-642 (2020).
102. Jung, J. et al. MRE combined with FIB-4 (MEFIB) index in detection of candidates for pharmacological treatment of NASH-related fibrosis. Gut 70 , 1946-1953 (2021).

103. Mosca, A., Panera, N., Crudele, A. \& Alisi, A. Noninvasive diagnostic tools for pediatric NAFLD: where are we now? Expert Rev. Gastroenterol. Hepatol. 14, 1035-1046 (2020).

104. Di Cesare, M. et al. Inequalities in non-communicable diseases and effective responses. Lancet 381 , 585-597 (2013).

105. Peters, R. et al. Common risk factors for major noncommunicable disease, a systematic overview of reviews and commentary: the implied potential for targeted risk reduction. Ther. Adv. Chronic Dis. 10, 2040622319880392-2040622319880392 (2019).

106. Fuchs, S., Henschke, C., Blümel, M. \& Busse, R. Disease management programs for type 2 diabetes in Germany: a systematic literature review evaluating effectiveness. Dtsch. Arztebl Int. 111 , 453-463 (2014).

107. World Health Organization. WHO package of essential noncommunicable (PEN) disease interventions for primary health care. WHO https://www.who.int/ publications/i/item/who-package-of-essentialnoncommunicable-(pen)-disease-interventionsfor-primary-health-care (2020).

108. Dixon, B. E., Embi, P. J. \& Haggstrom, D. A. Information technologies that facilitate care coordination: provider and patient perspectives. Transl. Behav. Med. 8, 522-525 (2018)

109. Ando, Y. \& Jou, J. H. Nonalcoholic fatty liver disease and recent guideline updates. Clin. Liver Dis. 17, 23-28 (2021).

110. Leoni, S. et al. Current guidelines for the management of non-alcoholic fatty liver disease: a systematic review with comparative analysis. World J. Gastroenterol. 24, 3361-3373 (2018).

111. European Association for the Study of the Liver (EASL); European Association for the Study of Diabetes (EASD); European Association for the Study of Obesity (EASO) EASL-EASD-EASO Clinical Practice Guidelines for the management of non-alcoholic fatty liver disease. J. Hepatol. 64 1388-1402 (2016).

112. Arab, J. P. et al. Latin American Association for the Study of the Liver (ALEH) practice guidance for the diagnosis and treatment of non-alcoholic fatty liver disease. Ann. Hepatol. 19, 674-690 (2020).

113. Chalasani, N. et al. The diagnosis and management of nonalcoholic fatty liver disease: practice guidance from the American Association for the Study of Liver Diseases. Hepatology 67, 328-357 (2018).

114. Chitturi, S. et al. The Asia-Pacific Working Party on Non-alcoholic Fatty Liver Disease guidelines 2017-Part 2: Management and special groups J. Gastroenterol. Hepatol. 33, 86-98 (2018).

115. Eslam, M. et al. The Asian Pacific Association for the Study of the Liver clinical practice guidelines for the diagnosis and management of metabolic associated fatty liver disease. Hepatol. Int. 14 889-919 (2020).

116. Muthiah, M. D. ¿ Sanyal, A. J. Current management of non-alcoholic steatohepatitis. Liver Int. $\mathbf{4 0}$ (Suppl. 1), 89-95 (2020).

117. Hallsworth, K. \& Adams, L. A. Lifestyle modification in NAFLD/NASH: facts and figures. JHEP Rep. Innov. Hepatol. 1, 468-479 (2019).

118. Romero-Gómez, M., Zelber-Sagi, S. \& Trenell, M. Treatment of NAFLD with diet, physical activity and exercise. J. Hepatol. 67, 829-846 (2017).

119. Jensen, M. D. et al. 2013 AHA/ACC/TOS guideline for the management of overweight and obesity in adults: a report of the American College of Cardiology/ American Heart Association Task Force on Practice Guidelines and The Obesity Society. Circulation 129 S102-S138 (2014).

120. Hydes, T. J., Ravi, S., Loomba, R. \& Gray, M. E. Evidence-based clinical advice for nutrition and dietary weight loss strategies for the management of NAFLD and NASH Clin Mol. Hepatol. 26, 383-400 (2020).

121. Geurtsen, M. L., Santos, S., Gaillard, R., Felix, J. F. $\&$ Jaddoe, V. W. V. Associations between intake of sugar-containing beverages in infancy with liver fat accumulation at school age. Hepatology 73, 560-570 (2021).

122. Berná, G. ¿ Romero-Gomez, M. The role of nutrition in non-alcoholic fatty liver disease: pathophysiology and management. Liver Int. 40 (Suppl. 1), 102-108 (2020). 
123. Hashida, R. et al. Aerobic vs resistance exercise in non-alcoholic fatty liver disease: a systematic review. J. Hepatol. 66, 142-152 (2017).

124. Lazarus, J. V. et al. A cross-sectional study of the public health response to non-alcoholic fatty liver disease in Europe. J. Hepatol. 72, 14-24 (2020).

125. Neuschwander-Tetri, B. A. Therapeutic landscape for NAFLD in 2020. Gastroenterology 158, 1984-1998. e3 (2020).

126. Wong, E. L. Y. et al. Patient experience and satisfaction with inpatient service: development of short form survey instrument measuring the core aspect of inpatient experience. PLOS ONE 10, e0122299 (2015).

127. Martin, L. R., Williams, S. L., Haskard, K. B. $\&$ Dimatteo, M. R. The challenge of patient adherence. Ther. Clin. Risk Manag. 1, 189-199 (2005).

128. World Health Assembly. Hepatitis. WHO https://apps. who.int/gb/ebwha/pdf_files/WHA67/A67_R6-en. pdf?ua $=1(2014)$

129. Puhl, R. M. \& Heuer, C. A. Obesity stigma: important considerations for public health. Am. J. Public Health 100, 1019-1028 (2010).

130. Room, R. Stigma, social inequality and alcohol and drug use. Drug Alcohol. Rev. 24, 143-155 (2005).

131. Vaughn-Sandler, V., Sherman, C., Aronsohn, A. \& Volk, M. L. Consequences of perceived stigma among patients with cirrhosis. Dig. Dis. Sci. 59, 681-686 (2014).

132. Himmelstein, M. S. \& Puhl, R. M. At multiple fronts: diabetes stigma and weight stigma in adults with type 2 diabetes. Diabet. Med. 38, e 14387 (2021).

133. Lazarus, J. V. et al. European 'NAFLD Preparedness Index'-is Europe ready to meet the challenge of fatty liver disease? JHEP Rep. 3, 100234 (2021).

134. Lazarus, J. V. et al. The global non-alcoholic fatty liver disease (NAFLD) preparedness index: are countries ready to tackle the challenge? Int. Liver Congr. 75 (Suppl. 2), 23-26 (2021).

135. NASH Council $\&$ Global Liver Institute. U.S NASH action plan (Global Liver Institute, 2020).

136. World Health Organization. Continuity and coordination of care: a practice brief to support implementation of the WHO Framework on integrated people-centred health services (Alliance for Health Policy and Systems Research, 2018)

137. Atun, R. et al. Improving responsiveness of health systems to non-communicable diseases. Lancet 381 690-697 (2013)

138. Rutter, H. et al. The need for a complex systems model of evidence for public health. Lancet 390 , 2602-2604 (2017)

139. Kumanyika, S., Jeffery, R. W., Morabia, A. Ritenbaugh, C. \& Antipatis, V. J. Obesity prevention: the case for action. Int. J. Obes. Relat. Metab. Disord. 26, 425-436 (2002)

140. Gortmaker, S. L. et al. Changing the future of obesity: science, policy, and action. Lancet $378,838-847$ (2011)

141. Huang, T. T., Drewnosksi, A., Kumanyika, S. \& Glass, T. A. A systems-oriented multilevel framework for addressing obesity in the 21 st century. Prev. Chronic Dis. 6, A82 (2009).

142. Huang, T. \& Ferris, E. in Childhood Obesity Ch. 42 (ed. Goran, M. I.) (Taylor and Francis, 2016).

143. Huang, T. T. et al. Mobilisation of public support for policy actions to prevent obesity. Lancet 385 2422-2431 (2015)

144. Lazarus, J. V. et al. A Sustainable Development Goal framework to guide multisectoral action on NAFLD through a societal approach. Preprint at medRxiv https://doi.org/10.1101/2021.08.02.21261464 (2021).

145. Adler, M. \& Ziglio, E. Gazing into the Oracle: The Delphi Method and its Application to Social Policy and Public Health (Jessica Kingsley, 1996).

146. Grime, M. M. \& Wright, G. in Wiley StatsRef: Statistics Reference Online 1-6 (Wiley, 2011).

147. Targher, G. \& Byrne, C. D. From nonalcoholic fatty liver disease to metabolic dysfunction-associated fatty liver disease: is it time for a change of terminology? Hepatoma Res. 6, 64 (2020)

148. Eslam, M. et al. A new definition for metabolic dysfunction-associated fatty liver disease: an international expert consensus statement. J. Hepatol. 73, 202-209 (2020).

149. Eslam, M., Sanyal, A. J. \& George, J. MAFLD a consensus-driven proposed nomenclature for metabolic associated fatty liver disease. Gastroenterology 158, 1999-2014.e1 (2020).

150. Mendez-Sanchez, N. et al. The Latin American Association for the Study of the Liver (ALEH) position statement on the redefinition of fatty liver disease. Lancet Gastroenterol. Hepatol. 6, 65-72 (2021).

151. Spearman, C. W. et al. The sub-Saharan Africa position statement on the redefinition of fatty live disease: from NAFLD to MAFLD. J. Hepatol. 74, 1256-1258 (2021).

152. Shiha, G. et al. Nomenclature and definition of metabolic-associated fatty liver disease: a consensus from the Middle East and north Africa. Lancet Gastroenterol. Hepatol. 6, 57-64 (2021).

153. Ratziu, V. et al. The times they are a-changin' (for NAFLD as well). J. Hepatol. 73, 1307-1309 (2020).

154. Younossi, Z. M. et al. From NAFLD to MAFLD: implications of a premature change in terminology. Hepatology 73, 1194-1198 (2021).

155. Wong, V. W. et al. Asia-Pacific Working Party on Non-alcoholic Fatty Liver Disease guidelines 2017-Part 1: definition, risk factors and assessment. J. Gastroenterol. Hepatol. 33, 70-85 (2018).

156. American Diabetes Association. Comprehensive medical evaluation and assessment of comorbidities: standards of medical care in diabetes - 2020 . Diabetes Care 43, S37-S47 (2020).

157. World Health Organization. Nothing for us, without us opportunities for meaningful engagement of people living with NCDs: meeting report (WHO, 2021).

\section{Acknowledgements}

The European Association for the Study of the Liver (EASL) International Liver Foundation (EILF) acknowledges fundin from Intercept Pharmaceutics, as well as Bristol-Myers Squibb and Merck Sharp \& Dohme. The authors thank in particular T. White (ISGlobal) and A. Hobbs (CUNY) for providing technical support during implementation of the Delph methodology, and to N. Lee and the Wilton Park team for facilitating the online convening of the core group on 16 February 2021. Three observers attended the session: M. Sicuro (EILF), Y. Nedelcheva (EASL) and K. Ray (Nature Reviews Gastroenterology \& Hepatology). The translations in Supplementary Tables 2-7 were provided by Springe Healthcare, and checked and revised by the authors, and are reproduced in Supplementary information as supplied.

\section{Author contributions}

J.V.L. and H.E.M. conceptualized the idea for the stud and developed the first draft of the consensus statements. The voting members of the core authorship group (Q.M.A. J.P.A., R.L.B. L.C., H.C.-P, J.C. K.C., M.A.D. S.F, J.G. H.H., T.T.-K.H., M.H.I., A.K., S.K.S., R.L., V.M., P.N.N., M.N., P.O V.R., M.R., M.R.-G., J.M.S., E.A.T., L.V., V.W.-S.W., Y.Y., Z.M.Y and S.Z.-S.) provided input on the draft statements ahead of Delphi round 1. D.R. supported the design of the study methodology, managed the Qualtrics XM platform and extracted data following each Delphi round. All members of the NAFLD Consensus Consortium participated in the Delphi study. J.V.L. and H.E.M. reviewed the data between Delphi rounds and revised the consensus statements based on the qualitative feedback. Core group members participated in the World Café discussion (J.V.L., H.E.M., Q.M.A., J.P.A., R.L.B., L.C., H.C.-P., J.C., S.F., H.H., T.T.-K.H., M.H.I., A.K., S.K.S., V.M P.N.N., M.N., P.O., M.R.-G., J.M.S., E.A.T., L.V., V.W.-S.W., Y.Y., Z.M.Y. and S.Z.-S.) prior to the third and final Delphi round J.V.L. and H.E.M. drafted the recommendations, which voting members of the authorship group reviewed and provided feedback on. All members of the NAFLD Consensus Consortium participated in at least one Delphi round. H.E.M and J.V.L. developed the first draft of the manuscript all authors contributed to and reviewed the full draft of the article, subsequent revisions, and approved the final version for submission.

\section{Competing interests}

A.K. declares grants/contracts from Intercept and Novartis and conference support from Intercept. E.A.T. has received personal fees from Intercept, Gilead, Pfizer and Promethera, and honoraria from Intercept, Gilead and Pfizer. E.A.T. is a member of the Governing Board of the European Association for the Study of the Liver. H.C.-P. has received honoraria from Intercept and Novo Nordisk. H.H. has received grants or contracts from Intercept, AstraZeneca, MSD, Pfizer, EchoSens, Stockholm City Council, Swedish Cancer Foundation, Radiumhemmets Forskningsfonder, Julins Fond, Stockholm Innovation Foundation, Skandia Research Foundation, Åke Wiberg Foundation, Bengt Ihre Foundation and Tore Nilson Foundation, participated on an advisory board for BMS and Gilead, and has stock or stock options in Novo Nordisk. H.E.M. has received grants to EASL International Liver
Foundation to support NAFLD activities from Intercept, Cenfit, BMS and MSD. J.C reports consultant and/or speaker and/or participated in clinical trials sponsored by and/or received grants and research support from Gilead Sciences, AbbVie, MSD, Shionogi, Intercept Pharmaceuticals, Janssen Pharmaceuticals, Celgene and Alexion (all outside the submitted work). J.M.S. declares a research grant from Gilead; consulting fees from Boehringer Ingelheim, BMS, Genfit and Gilead, Intercept Pharmaceuticals, Madrigal, Novartis, Novo Nordisk, Nordic Bioscience, Pfizer, Roche, Sanofi, Siemens Healthcare $\mathrm{GmbH}$; speakers bureau from Falk Foundation, and MSD Sharp \& Dohme. J.V.L. reports grants, personal fees and other support from AbbVie, personal fees from CEPHEID, personal fees from Genfit, grants, personal fees and other support from Gilead Sciences, personal fees from GSK, personal fees from Intercept, personal fees from Janssen, and grants and personal fees from MSD, outside the submitted work. L.C. declares: grants from Gilead; speaker's bureau for Abbvie, Echosens, Gilead, Intercept, Novo Nordisk; has been a member of an advisory board for Allergan, Alexion, Echosens, Gilead, Intercept, MSD, Novo Nordisk, Pfizer and Servier. LV declares grants or contracts from Gilead; honoraria from MSD, Gilead, AlfaSigma and Abbvie; support for attending conferences from Gilead; participation on advisory or safety boards for Intercept, Pfizer and Gilead. M.N. has received unrestricted research grants from Gilead, Abbie and Merck, and was President of the World Hepatitis Alliance 2018-2020. M.R. has received honoraria from Alnylam, Amgen, AMRA, BMS, Boehringer Ingelheim, Centara, Coheres, Enanta, Galecto, Intercept, Madrigal, NGM Biopharmaceuticals, Novo Nordisk, Pfizer, Fractyl, Gelesis, Siemens, Thetis, Terns, Rivus, 3vBio (Sagimet), 89bio, Novartis, Immuron, Merck and Taiwan J Pharmaceuticals. M.R.-G. reports grants from Intercept, grants from Gilead Sciences, personal fees from Shionogi, personal fees from Alfa Wassermann, personal fees from ProSciento, personal fees from Kaleido, personal fees from Novo Nordisk, personal fees from MSD, personal fees from BMS, personal fees from Allergan, personal fees from Boehringer Ingelheim, personal fees from Zydus, personal fees from Intercept Pharma and personal fees from Gilead Science, outside the submitted work. O.M.A. declares grant funding from multiple EFPIA partners via the EU IMI2-funded consortium Litmus (Abbvie, Antaros Medical, Allergan/Tobira, AstraZeneca, BMS, Boehringer Ingelheim International, Echosens, Ellegaard Gottingen Minipigs, Eli Lilly \& Company, Exalenz Bioscience Ltd., Genfit, Glympse Bio, GlaxoSmithKline, Histolndex, Intercept Pharma Europe Ltd., iXscient Ltd., Nordic Bioscience, Novartis Pharma AG, Novo Nordisk A/S, One Way Liver Genomics SL, Perspectum Diagnostics, Pfizer Ltd., Resoundant, Sanofi-Aventis Deutschland $\mathrm{GmbH}$, SomaLogic Inc., Takeda Pharmaceuticals International SA.); grants from Glympse Bio; royalties/licences from Elsevier; consulting fees from Allergan, AstraZeneca, Blade Therapeutics, BMS, Cirius Therapeutics, CymaBay, Eli Lilly, Galmed, Genfit, Gilead, Histolndex, Intercept, Inventiva, Madrigel, Metacrine, NGMBio, Novartis, Novo Nordisk, Pfizer, Poxel Pharma and The Medicines Company; honoraria from MedScape and Fishawack; and participation on safety or advisory boards for North Sea Therapeutics and Medpace. R.L.B. declares grants or contracts from Novo Nordisk and GLWL Research; consulting fees (personal) from Novo Nordisk, Viiv, Boehringer Ingelheim and International Medical Press; has participated on safety or advisory boards for Novo Nordisk and Pfizer; has been an unpaid trustee of the Association for the Study of Obesity, BOMMS Council Member and Trustee of Obesity Empowerment Network UK. R.L. declares grants or contracts from Allergan, AstraZeneca, Boehringer-Ingelheim, Bristol-Myers Squibb, Eli Lilly, Galectin Therapeutics, Galmed Pharmaceuticals, Genfit, Gilead, Intercept, Inventiva, Janssen, Madrigal Pharmaceuticals, Merck, NGM Biopharmaceuticals, Pfizer and Sonic Incytes; consulting fees from Aardvark Therapeutics, Altimmune, Anylam/Regeneron, Amgen, Arrowhead Pharmaceuticals, AstraZeneca, Bristol-Myers Squibb, CohBar, Eli Lilly, Galmed, Gilead, Glympse bio, Hightide, Inipharm, Intercept, Inventiva, Ionis, Janssen Inc., Madrigal, Metacrine, Inc., NGM Biopharmaceuticals, Novartis, Novo Nordisk, Merck, Pfizer, Sagimet, Theratechnologies, 89bio and Viking Therapeutics; and is co-founder of Liponexus. S.F. declares grants or contracts from Gilead, Roche, Bristol-Myers Squibb and Genfit; consulting fees from Roche, Gilead, Allergan, Abbvie, Bayer, Bristol-Myers Squibb, Boehringer Ingelheim, Merck Sharp \& Dohme, Janssen, Actelion, Astellas, Genfit, Inventiva, Intercept, Echosense, Genentech, Novo Nordisk, Novartis, AstraZeneca, Galmed, Promethera, Coherus, Madrigal, Julius Clinical, NGM Bio; and honoraria from Gilead, Genfit, Bayer, Abbvie, Intercept, Allergan. V.M. declares unrestricted contributions to the Liver Forum. V.R. declares grants or contracts from Gilead; consulting fees from Novo Nordisk, Galmed, Madrigal, AstraZeneca, Intercept, Terns, Theratechnologies, NGM, Bristol-Myers Squibb; and support for attending 
meetings from Gilead. V.W.-S.W. declares grants or contracts from Gilead (to institution); consulting fees and participation on boards (personal) from 3V-BIO, AbbVie, Allergan, Boehringer Ingelheim, Center for Outcomes Research in Liver Diseases, Echosens, Gilead Sciences, Hanmi Pharmaceutical, Intercept, Inventiva, Merck, Novartis, Novo Nordisk, Perspectum Diagnostics, Pfizer, ProSciento, Sagime Biosciences, TARGET PharmaSolutions and Terns; honoraria (personal) from Abbott, AbbVie, Bristol-Myers Squibb Echosens, Gilead Sciences; and support for attending meetings or travel (institution) from Abbvie and Gilead. Y.Y declares research grants from Biocordex and Gilead; honoraria from Gilead, Bilim, Pharmactive, Sanovel and Echosens; and participated on advisory boards for Novo Nordisk and Abbvie. J.P.A., K.C., M.A.D., J.G., T.T.-K.H
M.H.I., P.N.N., P.O., D.R., S.K.S., S.Z.-S. and Z.M.Y. declare no competing interests.

\section{Peer review information}

Nature Reviews Gastroenterology \& Hepatology thanks Anna Alisi, Rotonya Carr and the other, anonymous, reviewer(s) for their contribution to the peer review of this work

\section{Publisher's note}

Springer Nature remains neutral with regard to jurisdictional claims in published maps and institutional affiliations.

\section{Supplementary information}

The online version contains supplementary material available at https://doi.org/10.1038/s41575-021-00523-4.

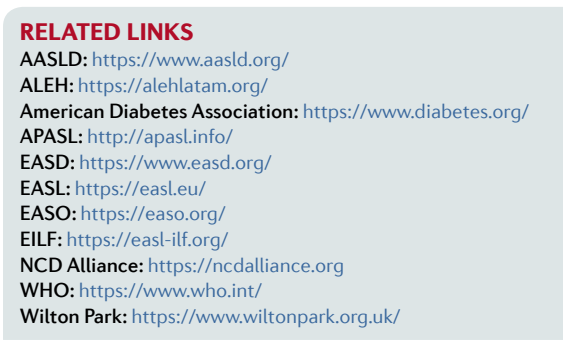

(C) Springer Nature Limited 2021, corrected publication 2021

\section{the NAFLD Consensus Consortium}

Jeffrey V. Lazarus ${ }^{1,2}$, Henry E. Mark², Quentin M. Anstee ${ }^{3,4}$, Juan Pablo Arab ${ }^{5}$, Rachel L. Batterham ${ }^{6}$, Laurent Castera ${ }^{7}$, Helena Cortez-Pinto ${ }^{8}$, Javier Crespo ${ }^{9}$, Kenneth Cusi $^{10}$, M. Ashworth Dirac ${ }^{11}$, Sven Francque ${ }^{12,13}$, Jacob George ${ }^{14}$, Hannes Hagström ${ }^{15}$, Terry T.-K. Huang ${ }^{16}$, Mona H. Ismail ${ }^{17}$, Achim Kautz ${ }^{18}$, Shiv Kumar Sarin ${ }^{19}$, Rohit Loomba ${ }^{20,21}$,

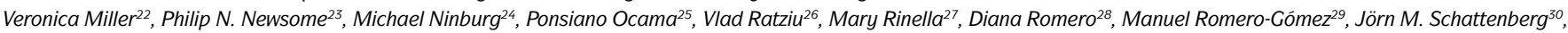
Emmanuel A. Tsochatzis ${ }^{31,32}$, Luca Valenti ${ }^{33,34}$, Vincent Wai-Sun Wong ${ }^{35}$, Yusuf Yilmaz ${ }^{36,37}$, Zobair M. Younossi38, Shira Zelber-Sagi ${ }^{39,40}$, Fredrik Åberg ${ }^{41,42}$, Leon Adams ${ }^{43}$, Maryam Salem Al Khatry ${ }^{44}$, Khalid Al Naamani45, Omar Alfaro Murillo, ${ }^{46}$, Alina M. Allen ${ }^{47}$, Faisal Alnaser ${ }^{48,49}$, Saleh A. Alqahtani ${ }^{50,51}$, Khalid Alswat ${ }^{52}$, Domenico Alvaro ${ }^{53}$, Raúl J. Andrade ${ }^{54,55}$, Marco Arrese ${ }^{5}$, Yaw Asante Awuku ${ }^{56}$, Motala Ayesha ${ }^{57}$, Oidov Baatarkhuu ${ }^{58}$, Shokhista Bakieva ${ }^{59}$, Rita Basu ${ }^{60}$, Ramon Bataller ${ }^{61}$, Shahinaz Bedri ${ }^{62}$, Emanuele Bosi ${ }^{63}$, Marc Bourliere ${ }^{64}$, Radan Bruha ${ }^{65}$, Elisabetta Bugianesi ${ }^{66}$, Patrizia Burra ${ }^{67}$, Maria Buti68, Christopher D. Byrne ${ }^{69}$, Jose Luis Calleja ${ }^{70}$, Patrizia Carrieri ${ }^{71}$, Flloyd Carter ${ }^{72}$, Marlen Ivon Castellanos Fernandez ${ }^{73}$, Gabriela Castillo-Lopez ${ }^{74}$, Graciela E. Castro-Narro ${ }^{75,76}$, Henry Lik Yuen Chan ${ }^{77}$, Wah-Kheong Chan ${ }^{78}$, Yoosoo Chang ${ }^{79}$, Massimo Colombo ${ }^{80}$, Kirsten J. Coppell ${ }^{81}$, Kathleen Corey ${ }^{82}$, Antonio Craxi ${ }^{83}$, Donna Cryer ${ }^{84}$, Anuradha Dassanayake ${ }^{85}$, Antonieta de Ascenção Soares Martins ${ }^{86}$, Victor de Ledinghen $^{87}$, Stefano DelPrato ${ }^{88}$, Alessandro Demaio ${ }^{89}$, Hailemichael Desalegn ${ }^{90}$, John Dillon ${ }^{91}$, Ajay Duseja9 ${ }^{92}$, Prabhakaran Dorairaj ${ }^{93}$, Mattias Ekstedt ${ }^{94}$, Mohamed El Kassas ${ }^{95}$, Osama M. Elsanousi ${ }^{96}$, Gamal Esmat ${ }^{97}$, Jian-Gao Fan ${ }^{98}$, Nathalie Farpour-Lambert ${ }^{99}$, Robert Flisiak ${ }^{100}$, Yasser Fouad ${ }^{101}$, Michael Fuchs ${ }^{102,103}$, Rino A. Gani ${ }^{104}$, Lynn Gerber ${ }^{105}$, Hasmik Ghazinyan 106, Liana Gheorghe ${ }^{107}$, George Boon-Bee Goh ${ }^{108}$, Henning Gronbcek ${ }^{109}$, Aghayeva Gulnara ${ }^{110}$, Saeed Hamid"11, Vanessa Hebditch ${ }^{12}$, Ingrid J. Hickman ${ }^{113}$, Samantha L. Hocking ${ }^{114}$, Bela Hunyady ${ }^{15}$, Ramazan Idilman ${ }^{116}$, Vasily A. Isakov ${ }^{177}$, Mohammad H. Jamal ${ }^{118}$, Peter Jepsen ${ }^{109}$, Natacha Jreige Iskandar ${ }^{119}$, Myeong Jun Song ${ }^{120,}$ K. C. Sudhamshu'121, Satoru Kakizaki ${ }^{12}$, George Kalamitsis ${ }^{123}$, Fasiha Kanwal'124, Jia-Horng Kao ${ }^{125}$, Lee Kaplan ${ }^{82}$, Takumi Kawaguchi'26, Yousef Khader ${ }^{127}$, Seung Up Kim ${ }^{128}$, Nicolas Kodjoh ${ }^{129}$, Ger Koek ${ }^{130}$, Kazuhiko Koike ${ }^{131}$, Narcisse Patrice Komas ${ }^{132}$, Marko Korenjak ${ }^{133}$, Marcelo Kugelmas ${ }^{134}$, Asma Labidi' ${ }^{135}$, Naomi F. Lange ${ }^{136}$, Joel E. Lavine' ${ }^{137}$,

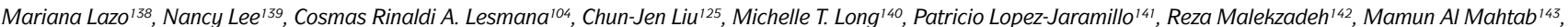
Giulio Marchesini'14, Rui Marinho ${ }^{145}$, Sophia E. Martínez Vázquez ${ }^{76}$, Lyudmila Mateva146, Charles Mbendi Nlombi ${ }^{147}$, Pascal Melin ${ }^{148}$, Ivana Mikolasevic 149, Tamara Milovanovic ${ }^{150}$, Carla Musso ${ }^{151}$, Atsushi Nakajima ${ }^{152}$, Edna Nava ${ }^{153}$, Alexander V. Nersesovi ${ }^{154}$, Dafina Nikolova ${ }^{155}$, Suzanne Norris ${ }^{156}$, Katja Novak ${ }^{157}$, Jude Oben ${ }^{158}$, Janus P. Ong ${ }^{159}$, Charles Onyekwere ${ }^{160}$, George Papatheodoridis ${ }^{161}$, Imran Paruk ${ }^{162}$, Keyur Patel ${ }^{163}$, M. Paula Macedo ${ }^{164}$, Carlos Penha-Gonçalves ${ }^{165}$, Marlene Pérez Figueroa ${ }^{166}$, Wolf Peter

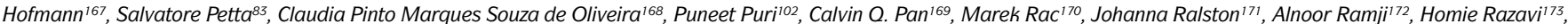
Mario Reis Alvares-da-Silva174, Stuart Roberts 175, Michael Roden 176, 177,178, Tamsin Rose179, Samir Rouabhia'180, Patrizia Rovere-Querini'18, Ian A. Rowe 182, Shakhlo Sadirova ${ }^{59}$, Riina Salupere ${ }^{183}$, Tobokalova Saparbu ${ }^{184}$, Raymond Sayegh ${ }^{185}$, Giada Sebastiani ${ }^{186}$, Yosuke Seki ${ }^{187}$, Josefina Selmo ${ }^{188}$, Abdel Karim Serme ${ }^{189}$, Jonathan E. Shaw ${ }^{190}$, Thrivikrama Shenoy ${ }^{191}$, Nick Sheron ${ }^{192}$, Oren Shibolet ${ }^{193}$, Marcelo Silva ${ }^{194}$, Igor Skrypnyk ${ }^{195}$, Piotr Socha ${ }^{196}$, Joan Soriano ${ }^{197}$, C. Wendy Spearman ${ }^{198}$, Kannan Sridharan ${ }^{199}$, Juan José Suárez ${ }^{200}$, Dhastagir Sultan Sheriffeol, Ki-Chul Sung ${ }^{202}$, Mark Swain ${ }^{203}$, Frank Tacke ${ }^{204}$, Shahrad Taheri205, Soek-Siam Tan ${ }^{206}$, Elliot B. Tapper ${ }^{207}$, Hannele Yki-Järvinen ${ }^{42,208,209}$, Maja Thiele ${ }^{210,211}$, Isaac Thom Shawa212, leva Tolmane 213,214 , Esther A. Torres ${ }^{215}$, Michael Trauner216, Sombat Treeprasertsuk ${ }^{217}$, Adela Turcanu ${ }^{218}$, Jonas Valantinas ${ }^{219}$, Mette Vesterhus ${ }^{220}$, Imam Waked ${ }^{221}$, Sarah H. Wild ${ }^{222}$, Jose Willemse ${ }^{223}$, Robert J. Wong ${ }^{224}$, Stavra Xanthakos ${ }^{225,226}$, Dan Yock Young ${ }^{227}$, Ming-Lung Y Y228, Kenneth I. Zheng ${ }^{229}$ Mudjat Zeybel230 and Ming-Hua Zheng ${ }^{229}$

${ }^{41}$ Transplantation and Liver Surgery, Helsinki University Hospital, Helsinki, Finland. ${ }^{42}$ University of Helsinki, Helsinki, Finland. ${ }^{43}$ University of Western Australia, Perth, Western Australia, Australia. ${ }^{44} \mathrm{l}$ brahim Bin Hamad Obaid Allah Hospital, Ras al Khaimah, United Arab Emirates. ${ }^{45}$ Armed Forces Hospital, Muscat, Oman. ${ }^{46}$ Association of Specialists in Gastroenterology and Digestive Endoscopy of Costa Rica, San Jose, Costa Rica. ${ }^{47}$ Mayo Clinic, Rochester, NY, USA. ${ }^{48}$ Imperial College London, London, UK. ${ }^{49} \mathrm{Home}$ Health Care Centre, Maqabah, Bahrain. ${ }^{50}$ Liver Transplant Centre, King Faisal Specialist Hospital and Research Centre, Riyadh, Saudi Arabia. ${ }^{51}$ Division of Gastroenterology \& Hepatology, Johns Hopkins University, Baltimore, MD, USA. ${ }^{52}$ Liver Disease Research Center, College of Medicine, King Saud University, Riyadh, Saudi Arabia. ${ }^{53}$ Sapienza University of Rome, Rome, Italy. ${ }^{54}$ UGC Aparato Digestivo and Servicio de Farmacología Clínica, Instituto de Investigaciōn Biomédica de Málaga - IBIMA, Hospital Universitario Virgen de la Victoria, Universidad de Mălaga, Málaga, Spain. ${ }^{55}$ Centro de Investigaciōn Biomédica en Red de Enfermedades Hepáticas y Digestivas (CIBERehd), Madrid, Spain. ${ }^{56}$ University of Health and Allied Sciences, Ho, Ghana. ${ }^{57}$ University of KwaZulu-Natal, Durban, South Africa. ${ }^{58}$ Mongolian National University of Medical Sciences, Ulaanbaatar, Mongolia. ${ }^{59}$ Research Institute of Virology of the Ministry of Health of the Republic of Uzbekistan, Tashkent, Uzbekistan. ${ }^{60}$ University of Virginia, Charlottesville, VA, USA. ${ }^{61}$ University of Pittsburgh, Pittsburgh, PA, USA. ${ }^{62}$ National Public Health Laboratory of Sudan and Ahfad University for Women, Khartoum, Sudan. ${ }^{63}$ Diabetes Research Institute, San Raffaele Hospital and San Raffaele Vita Salute University, Milan, Italy. ${ }^{64}$ Saint Joseph Hospital Marseille, Marseille, France. ${ }^{65}$ General University Hospital and Charles University, Prague, Czech Republic. ${ }^{66}$ Department of Medical Sciences, University of Turin, Turin, Italy. ${ }^{67}$ University of Padua-Italy, Padua, Italy. ${ }^{68}$ Vall d'Hebron University Hospital, Barcelona, Spain. ${ }^{69}$ Southampton National Institute for Health Research Biomedical Research Centre and the Institute of Developmental Sciences, University of Southampton, Southampton, UK. ${ }^{70} \mathrm{Hospital}$ University of Puerta de Hierro, Majadahonda, Spain. ${ }^{71}$ Aix Marseille Univ, Inserm, IRD, SESSTIM, Sciences Economiques \& Sociales de la Santé \& Traitement de l'Information Médicale, ISSPAM, Marseille, France. ${ }^{72}$ University of the West Indies, Nassau, Bahamas, ${ }^{73}$ Institute of Gastroenterology, Medical University of Havana, Havana, Cuba, ${ }^{74}$ Buenos Aires University, Buenos Aires, Argentina. ${ }^{75}$ Latin American Association for the Study of the Liver (ALEH), Mexico City, Mexico. ${ }^{76}$ Gastroenterology Department, Instituto Nacional de Ciencias Médicas y Nutrición Salvador Zubirán, Mexico City, Mexico. ${ }^{77}$ Union Hospital, Hong Kong, China. ${ }^{78}$ Gastroenterology and Hepatology Unit, Department of Medicine, Faculty of Medicine, University of Malaya, Kuala Lumpur, Malaysia. ${ }^{79}$ Center for Cohort Studies, Total Healthcare Center, Kangbuk Samsung Hospital, Sungkyunkwan University School of Medicine, Seoul, Republic of Korea. ${ }^{80}$ San Raffaele Hospital, Milan, Italy. ${ }^{81}$ Department of Medicine, University of Otago, Dunedin, New Zealand. ${ }^{82}$ Massachusetts General Hospital and Harvard Medical School, Boston, MA, USA. ${ }^{83}$ University of Palermo, Palermo, Italy. ${ }^{84} \mathrm{Global}$ Liver Institute, Washington, DC, USA. ${ }^{85}$ University of Kelaniaya, Colombo, Sri Lanka. ${ }^{86}$ University of Cabo Verde, Praia, Cabo Verde. ${ }^{87} \mathrm{Hospital}$ Center University of Bordeaux, Bordeaux, France. ${ }^{88}$ University of Pisa, Pisa, Italy. ${ }^{89}$ VicHealth, Melbourne, Victoria, Australia. ${ }^{90}$ St. Paul's Hospital Millennium Medical College, Addis Ababa, Ethiopia. ${ }^{91}$ University of Dundee, Dundee, UK. ${ }^{92}$ Postgraduate Institute of Medical Education and Research, Chandigarh, India. ${ }^{93}$ World Heart Federation, Geneva, Switzerland. ${ }^{94}$ Department of Health, Medicine and Caring Sciences, Linköping University, Linköping, Sweden. ${ }^{95}$ Endemic Medicine Department, Faculty of Medicine, Helwan University, Cairo, Egypt. ${ }^{96}$ The National Ribat University, Khartoum, Sudan. ${ }^{97}$ Cairo University Hospitals, Cairo, Egypt. ${ }^{98}$ Department of Gastroenterology, Xinhua Hospital, Shanghai Jiao Tong University School of Medicine, Shanghai, China. ${ }^{99}$ European Association for the Study of Obesity (EASO), Teddington, UK, ${ }^{100}$ Medical University of Bialystok. Bialystok, Poland ${ }^{101}$ Minia University, Minia, Egypt ${ }^{102}$ Virginia Commonwealth University and Central Virginia Veterans Affairs Health Systems, Richmond, VA, USA. ${ }^{103}$ Virginia Commonwealth University, Division of Gastroenterology, Hepatology and Nutrition, Richmond, VA, USA. ${ }^{104 H e p a t o b i l i a r y}$ Division, Department of Internal Medicine, Dr. Cipto Mangunkusumo National General Hospital, Universitas Indonesia, Jakarta, Indonesia. ${ }^{105}$ Inova Health System, Falls Church, VA, USA. ${ }^{106}$ Armenian Association for the Study of the Liver (ARASL), Yerevan, Armenia. ${ }^{107}$ University of Medicine and Pharmacy Carol Davila, Bucharest, Romania. ${ }^{108}$ Department of Gastroenterology \& Hepatology, Singapore General Hospital, Singapore, Singapore. ${ }^{109}$ Department of Hepatology and Gastroenterology, Aarhus University Hospital, Aarhus, Denmark. ${ }^{110}$ Azerbaijan Gastroenterologists and Hepatologists Association, Baku, Azerbaijan. ${ }^{111}$ Aga Khan University, Karachi, Pakistan. ${ }^{112}$ British Liver Trust, Bournemouth, UK. ${ }^{113}$ Princess Alexandra Hospital, Brisbane, Queensland, Australia. ${ }^{114}$ Department of Endocrinology, Royal Prince Alfred Hospital and Central Clinical School, Faculty of Medicine and Health, University of Sydney, Sydney, New South Wales, Australia. ${ }^{115}$ Somogy County Kaposi Môr Teaching Hospital, Kaposvăr and University of Pécs, Pécs, Hungary. ${ }^{116}$ Ankara University School of Medicine, Ankara, Turkey. ${ }^{117}$ Department of Gastroenterology \& Hepatology, Federal Research Center of Nutrition, Biotechnology \& Food Safety, Moscow, Russia. ${ }^{118}$ Kuwait University, Kuwait City, Kuwait. ${ }^{119}$ Horacio Oduber Hospital, Oranjestad, Aruba. ${ }^{120}$ Department of Internal Medicine, College of Medicine, The Catholic University of Korea, Seoul, Republic of Korea. ${ }^{121}$ National Academy of Medical Sciences, Kathmandu, Nepal. ${ }^{122}$ Department of Gastroenterology, National Hospital Organization Takasaki General Medical Center, Takasaki, Japan. ${ }^{123}$ Liver Patients International, Athens, Greece. ${ }^{124}$ Baylor College of Medicine, Houston, TX, USA. ${ }^{125}$ National Taiwan University Hospital, Taipei, Taiwan. ${ }^{126}$ Division of Gastroenterology, Department of Medicine, Kurume University School of Medicine, Kurume, Japan. ${ }^{127}$ Jordan University of Science and Technology, Irbid, Jordan. ${ }^{128}$ Yonsei University, Seoul, Republic of Korea. ${ }^{129}$ University of Abomey Calavi, Faculty of Health Sciences, Cotonou, Benin. ${ }^{130}$ Department of Internal Medicine, Division of Gastroenterology and Hepatology, Maastricht University Medical Center, Maastricht, Netherlands. ${ }^{131}$ University of Tokyo, Tokyo, Japan. ${ }^{132}$ Pasteur Institute of Bangui, Bangui, Central African Republic. ${ }^{133}$ European Liver Patients Association, Brussels, Belgium. ${ }^{134}$ South Denver Gastroenterology, Denver, CO, USA. ${ }^{135}$ Gastroenterology "A" 


\section{CONSENSUS STATEMENT}

Department, Rabta Hospital, Faculty of Medicine of Tunis, Tunis, Tunisia. ${ }^{136}$ University Clinic for Visceral Surgery and Medicine, Inselspital Bern, Bern, Switzerland. ${ }^{137}$ Columbia University, New York, NY, USA ${ }^{138}$ Dornsife School of Public Health, Drexel University, Philadelphia, PA, USA. 139 Wilton Park, Steyning. UK. ${ }^{140}$ Boston University School of Medicine, Boston, MA, USA. ${ }^{141}$ University of Santander (UDES), Bucaramanga, Colombia. ${ }^{142}$ Digestive Disease Research Center, Tehran University of Medical Science, Tehran, Iran. ${ }^{143}$ Bangabandhu Sheikh Mujib Medical University, Dhaka, Bangladesh. ${ }^{144}$ University of Bologna, Bologna, Italy. ${ }^{145}$ Lisbon North University Hospital Center, Lisbon, Portugal. ${ }^{146}$ University Hospital St. Ivan Rilski, Sofia, Bulgaria. ${ }^{147}$ Service of Hepatogastroenterology, Department of Internal Medicine, University Clinic of Kinshasa, University of Kinshasa, Kinshasa, Democratic Republic of the Congo. ${ }^{148}$ Saint-Dizier Hospital Center, Saint Dizier, France. ${ }^{149}$ University Hospital Center Rijeka, Rijeka, Croatia. ${ }^{150}$ Clinical Center of Serbia, Beograd, Serbia. ${ }^{151}$ Favaloro Foundation, Buenos Aires, Argentina. ${ }^{152}$ Yokohama City University, Yokohama, Japan. ${ }^{153}$ University of Nuevo Leon, San Nicolás de los Garza, Mexico. ${ }^{154}$ Asfendiyarov Kazakh National Medical University, Almaty, Kazakhstan. ${ }^{155}$ University Clinic of Gastroenterohepatology, University Ss. Cyril and Methodius, Skopje, Republic of North Macedonia. ${ }^{156}$ St James's Hospital, Dublin, Ireland. ${ }^{157}$ University Medical Center Ljubljana, Ljubljana, Slovenia. ${ }^{158} \mathrm{Guy}$ 's and St Thomas' Hospital, London, UK. ${ }^{159}$ University of the Philippines College of Medicine, Manila, Philippines. ${ }^{160}$ Lagos State University College of Medicine, Lagos, Nigeria. ${ }^{161}$ National and Kapodistrian University of Athens, Athens, Greece. ${ }^{162}$ University of KwaZulu Natal, Durban, South Africa. ${ }^{163}$ University Health Network Toronto, Toronto, Ontario, Canada. ${ }^{164}$ Centro de Estudos de Doenças Crónicas (CEDOC), NOVA Medical School-FCM, Universidade Nova de Lisboa, Lisboa, Portugal. ${ }^{165}$ Instituto Gulbenian de Ciencia, Oeiras, Portugal. ${ }^{166}$ Dominican Society of Gastroenterology, Santo Domingo, Dominican Republic. ${ }^{167}$ Gastroenterology at Bayerischer Platz, Berlin, Cermany. 168 University of Sao Paulo School of Medicine, Sao Paulo, Brazil. ${ }^{169}$ NYU Langone Health, NYU Grossman School of Medicine, New York, NY, USA. ${ }^{170}$ Teaching Hospital Nitra, Nitra, Slovakia. ${ }^{171}$ World Obesity Federation, London, UK. ${ }^{172}$ University of British Columbia, Vancouver, British Columbia, Canada. ${ }^{173}$ Center for Disease Analysis Foundation, Lafayette, LA, USA. ${ }^{174}$ School of Medicine, Universidade Federal do Rio Grande do Sul, Porto Alegre, Brazil. ${ }^{175}$ Alfred Health, Melbourne, Victoria, Australia. ${ }^{176}$ Department of Endocrinology and Diabetology, Medical Faculty and University Hospital, Heinrich-Heine University, Düsseldorf, Germany. ${ }^{177}$ Institute for Clinical Diabetology, German Diabetes Center, Leibniz Center for Diabetes Research at Heinrich-Heine University, Düsseldorf, Germany. ${ }^{178}$ German Center for Diabetes Research (DZD), Partner Düsseldorf, Düsseldorf, Germany. ${ }^{179}$ Friends of Europe, Brussels, Belgium. ${ }^{180}$ University Hospital Touhami Benflis, Batna, Algeria. ${ }^{181}$ IRCCS San Raffaele Hospital and University, Milan, Italy. ${ }^{182}$ University of Leeds, Leeds, UK. ${ }^{183}$ University of Tartu, Tartu University Hospital, Tartu, Estonia. ${ }^{184}$ Kyrgyz State Medical Institute for Advanced Training and Retraining, Bishkek, Kyrgyz Republic. ${ }^{185} \mathrm{Hotel}$ Dieu de France and St Joseph University School of Medicine, Beirut, Lebanon. ${ }^{186}$ McGill University Health Centre, Montreal, Quebec, Canada. ${ }^{187}$ Weight LosS and Metabolic Surgery Center, Yotsuya Medical Cube, Tokyo, Japan. ${ }^{188}$ Ciudad Sanitaria Dr. Luis E. Aybar, Santo Domingo, Dominican Republic. ${ }^{189}$ University of Ouagadougou, Ouagadougou, Burkina Faso. ${ }^{190}$ Baker Heart and Diabetes Institute, Melbourne, Victoria, Australia. ${ }^{191}$ Sree Gokulam Medical College, Venjarammoodu, Kerala, India. ${ }^{192}$ Kings College London, London, UK. ${ }^{193}$ Tel-Aviv Medical Centre and Tel-Aviv University, Tel-Aviv, Israel. ${ }^{194}$ Austral University Hospital, Pilar, Argentina. ${ }^{195}$ Poltava State Medical University, Poltava, Ukraine. ${ }^{196}$ The Children's Memorial Health Institute, Warsaw, Poland. ${ }^{197} \mathrm{La}$ Princesa University Hospital, Madrid, Spain. ${ }^{198}$ Division of Hepatology, Department of Medicine, Faculty of Health Sciences, University of Cape Town, Cape Town, South Africa. ${ }^{199}$ Department of Pharmacology \& Therapeutics, College of Medicine \& Medical Sciences, Arabian Gulf University, Manama, Kingdom of Bahrain. ${ }^{200}$ Universidad San Francisco de Quito, Medicine School, Quito, Ecuador. ${ }^{201}$ Anna Medical College, Monte Blanche, Mauritius. ${ }^{202}$ Kangbuk Samsung Hospital, Sungkunkwan University, Seoul, Republic of Korea. ${ }^{203}$ University of Calgary, Calgary, Alberta, Canada. ${ }^{204}$ Charitê Universitätsmedizin Berlin, Berlin, Germany. ${ }^{205}$ Weill Cornell Medicine Qatar, Ar-Rayyan, Qatar. ${ }^{206}$ Selayang Hospital, Batu Caves, Malaysia. ${ }^{207}$ University of Michigan, Ann Arbor. MI, USA. ${ }^{208} \mathrm{Helsinki}$ University Hospital, Helsinki, Finland. ${ }^{209}$ Minerva Foundation Institute for Medical Research, Helsinki, Finland. ${ }^{210}$ Odense University Hospital, Odense, Denmark. ${ }^{211}$ University of Southern Denmark, Odense, Denmark. ${ }^{212}$ University of Malawi College of Medicine, Blantyre, Malawi. ${ }^{213}$ Riga East University Hospital, Riga, Latvia. ${ }^{214}$ University of Latvia, Riga, Latvia. ${ }^{215}$ University of Puerto Rico School of Medicine, San Juan, Puerto Rico. ${ }^{216}$ Medical University of Vienna, Vienna, Austria. ${ }^{217}$ King Chulalongkorn Memorial Hospital and Chulalongkorn University, Bangkok, Thailand. ${ }^{218}$ Gastroenterology, State University of Medicine and Pharmacy "Nicolae Testemitanu”, Chișinău, Republic of Moldova. ${ }^{219}$ Vilnius University Hospital Santaros Clinics, Vilnius, Lithuania. ${ }^{220} \mathrm{Haraldsplass}$ Deaconess Hospital and University of Bergen, Bergen, Norway. ${ }^{221}$ National Liver Institute, Shibin Al Kawm, Egypt. ${ }^{222}$ Usher Institute, University of Edinburgh, Edinburgh, UK. ${ }^{223}$ Dutch Liver Patients Association, Hoogland, Netherlands. ${ }^{224}$ Stanford University School of Medicine, Stanford, CA, USA. ${ }^{225}$ Cincinnati Children's Hospital Medical Center, Cincinnati, OH, USA. ${ }^{226}$ University of Cincinnati College of Medicine, Cincinnati, OH, USA. ${ }^{227} \mathrm{National}$ University, Singapore, Singapore. ${ }^{228}$ Hepatobiliary Section, Department of Internal Medicine, Kaohsiung Medical University Hospital, Kaohsiung Medical University, Kaohsiung, Taiwan. ${ }^{229}$ NAFLD Research Center, Department of Hepatology, the First Affiliated Hospital of Wenzhou Medical University, Wenzhou, China. ${ }^{230}$ Koç Üniversitesi, Istanbul, Turkey. 